INSTITUT NATIONAL DE RECHERCHE EN INFORMATIQUE ET EN AUTOMATIQUE

\title{
Discrete version of the She asymtpotics: multigroup neutron transport equations
}

\author{
Thierry Goudon — Antoine Mellet
}

\section{$\mathbf{N}^{\circ} 4302$}

novembre 2001

THÈME 4 



\title{
Discrete version of the She asymtpotics: multigroup neutron transport equations
}

\author{
Thierry Goudon*, Antoine Mellet ${ }^{\dagger}$ \\ Thème 4 - Simulation et optimisation \\ de systèmes complexes \\ Projet Caiman \\ Rapport de recherche $n^{\circ} 4302$ - novembre 2001 - 37 pages
}

\begin{abstract}
This paper is devoted to the derivation of multigroup diffusion equations from the Boltzmann equation. The limit system couples the energy levels from both zeroth order term and diffusion currents.

Key-words: Boltzmann equation, Diffusion approximation, Neutron transport, Spherical Harmonics Expansion, Transport-diffusion equation
\end{abstract}

AMS Subject classification: $35 \mathrm{Q} 20,82 \mathrm{~A} 70,76 \mathrm{P} 05,78 \mathrm{~A} 35,41 \mathrm{~A} 60$

* Labo. J.A. Dieudonné UMR 6621, Université Nice-Sophia Antipolis, Parc Valrose, F-06108 Nice cedex 02, France, et INRIA project CAIMAN, BP 93, 06902 Sophia Antipolis Cédex, France, goudon@math.unice.fr

$\dagger$ Mathématiques pour l'Industrie et la Physique, UMR 5640, Université Paul Sabatier, 118, route de Narbonne, F-31062 Toulouse cedex, France, mellet@mip.ups-tlse.fr 


\section{Une version discrète des asymptotiques de She : équations multigroupes de transport de neutrons}

Résumé : Ce rapport est consacré à l'obtention des équations de diffusion multigroupe à partir des équations de Boltzmann. Le système limite couple les niveaux d'énergie dès le niveau zéro et les courants de diffusion.

Mots-clés : Equation de Boltzmann, approximation par diffusion, transport de neutron, harmoniques sphériques, équations de convection-diffusion 


\section{Introduction}

Production of nuclear energy relies on the disintegration of atoms (Uranium or Plutonium), when subjected to collisions with neutrons. Therefore, reactor design requires an accurate description of the motion of the population of neutrons. The motion of neutrons is described through the evolution of the density $f(t, x, v)$ of neutrons occupying at time $t \geq 0$ the position $x \in \mathbb{R}^{N}$ and having velocity $v \in \mathbb{R}^{N}$. It will be convenient in what follows to see this last variable as $v=\sqrt{2 e / m} \omega, m>0$ being the mass of the neutron, $e \geq 0$ its energy and $\omega \in S^{N-1}$ the direction of the flight. The unknown $f$ verifies a transport equation

$$
\partial_{t} f+v \cdot \nabla_{x} f=Q(f)
$$

that relates the free transport (left hand side) to the various interaction processes undergone by the neutrons and described through the operator $Q(f)$. The latter are essentially collisions. Furthermore, the number of neutrons in any volume, at any time, remains smaller than the number of atomic nuclei $\left(10^{11}\right.$ is a typical ratio) so that it is reasonable to assume that the most probable event is an elastic collision with the surrounding medium. Consequently, the right hand side of (1) is usually given by a Boltzmann linear operator

$$
Q(f)=\int \sigma\left(x, v, v^{\prime}\right) f\left(t, x, v^{\prime}\right) d v^{\prime}-\Sigma(x, v) f(t, x, v) .
$$

The transfer function $\sigma\left(x, v, v^{\prime}\right) \geq 0$ is such that $\sigma\left(x, v, v^{\prime}\right) d v$ represents the probability that a neutron impinging with velocity $v^{\prime}$ will have velocity in the volume $d v$ around $v$ after the collision; while $\Sigma(x, v) \geq 0$ is the so-called removal cross section. If the operator is conservative i.e. $\int Q(f) d v=0$, which means that $\Sigma(x, v)=\int \sigma\left(x, v^{\prime}, v\right) d v^{\prime}$, then absorption/fission events are neglected or compensated with scattering. One also says that the reactor is critical in this case. It is worth remarking that, since the ratio of the mass of the nuclei to the mass of the neutrons is very large, then, during an elastic collision, the energy of the neutron is practically unchanged; the main effect of such a collision is only to modify the direction of the flight. Notice also that inelastic scattering, with loss of energy, remains possible, but it can only occur for highly energetic neutrons.

On the other hand, nuclear engineers are motivated in the derivation of simplified models that describe the physics accurately enough but remain of moderate computational cost. In these applications, one encounters a very large range of energies, from $1 / 40 \mathrm{eV}$ to some $M e v$; and a first simplification arises by breaking the energy range into several disjoint energy groups: $\left[e_{\min }, e_{M a x}\right]=\bigcup_{i=1}^{I}\left[e_{i}, e_{i+1}[\right.$. In most of the situations, the total number of groups $I$ is finite. One assumes that the cross sections do not vary too much on the energy groups and neutrons evolve according to averaged quantities, such as average scattering cross sections. Consequently, one is led to semi-discrete versions of equation (1-2). This will be detailed in Section 2 below.

Next, a commonly used strategy consists in neglecting the angular variable $\omega$ and writing a diffusion equation for a macroscopic energy distribution function $\rho(t, x, e), e$ being the energy variable (discrete or continuous). Actually, one deals in this context with systems of 
equations, coupled by the energy variable, which have usually the following form

$$
\begin{aligned}
\partial_{t} \rho(t, x, e)- & \operatorname{div}_{x}\left(D(x, e) \nabla_{x} \rho(t, x, e)\right) \\
& =\int \widetilde{\sigma}\left(x, e, e^{\prime}\right) \rho\left(t, x, e^{\prime}\right) d e^{\prime}-\widetilde{\Sigma}(x, e) \rho(t, x, e),
\end{aligned}
$$

where the coefficients $D, \widetilde{\sigma}, \widetilde{\Sigma}$ are non-negative. Of course, one obtains similar systems in the (energy-)discretized context. These equations can be derived directly from a balance relation on the population of neutrons, through a phenomenological analysis of the scattering events. The diffusion coefficients are due to elastic scattering when neutrons do not lose energy during the collision process. Zeroth order terms are due to absorption, inelastic scattering (i.e. collisions with loss of energy) or fission. Furthermore, system (3) can be generalized by postulating that the diffusion operator couples the energy levels as follows

$$
\operatorname{div}_{x}\left(\int k\left(x, e, e^{\prime}\right) \nabla_{x} \rho\left(t, x, e^{\prime}\right) d e^{\prime}\right) .
$$

Indeed, gradients of the density $\rho$ at a given energy $e^{\prime}$ can have some effect on the diffusion on another energy level $e$.

However, it is also tempting to obtain systems like (3) from the kinetic modeling, eq. (1-2), and to identify the coefficients through an asymptotic analysis. Then, starting from the continuity equation

$$
\partial_{t} \rho+\operatorname{div}_{x} j=\int_{S^{N-1}} Q(f) d \omega
$$

satisfied by the macroscopic density

$$
\rho(t, x, e)=\int_{S^{N-1}} f(t, x, \sqrt{2 e / m} \omega) d \omega,
$$

and the macroscopic current

$$
j(t, x, e)=\int_{S^{N-1}} \sqrt{2 e / m} \omega f(t, x, \sqrt{2 e / m} \omega) d \omega,
$$

we search for a diffusion equation by postulating a relation between $\rho$ and $j$ that takes the form of a generalized Fick's law

$$
j(t, x, e)=-\int k\left(x, e, e^{\prime}\right) \nabla_{x} \rho\left(t, x, e^{\prime}\right) d e^{\prime} .
$$

Such an approximation is intended to apply in the limit of small mean free paths, which is related to the probability of collisions by unit length and decreases when the density of the medium increases. It leads to singular perturbation problems and this work is devoted to such a question.

We refer for details on the physics of nuclear reactors to the classical books of B. Davison [17], E. Wigner [41], E. Wachspress [40], J. Bussac-P. Reuss [12], J. Planchard [37], C. 
Cercignani [15]. The mathematical study of the vanishing mean free path limit and of the diffusion approximation is by now a classical problem with applications in various fields of physics; we refer among others to E. Larsen-J. Keller [32], A. Bensoussan-J.-L. LionsG. Papanicolaou [10], C. Bardos-R. Santos-R. Sentis [8] and, for recent progresses, to R. Dautray-J.L. Lions [16], F. Malvagi-D. Levermore-G. Pomraning [35], F. Poupaud [38], F. Golse [26], C. Bardos-F. Golse-B. Perthame [6], C. Bardos-F. Golse-B. Perthame-R. Sentis [7], F. Golse-F. Poupaud [27], P. L. Lions-G. Toscani [34] P. Degond-T. Goudon-F. Poupaud [20]... Another difficulty in reactor physics is related to the high heterogeneity of the surrounding medium. Accordingly, the cross sections depend on the space variable and present very large oscillations. This leads to homogenization questions. Depending on the ordering, the homogenization procedure can be performed at the kinetic level, see F. Golse [24, 25], L. Dumas-F. Golse [22], P. Gérard-F. Golse [23] or directly on the diffusion approximation as in J. Dorning-R. Uddin-H. Zhang [21] Y. Capdeboscq [13, 14], G. AllaireY. Capdeboscq [2], G. Allaire-F. Malige [3]. We can also combine altogether these effects as in E. Larsen [30, 31], E. Larsen-M. Williams [33], C. Bardos-L. Dumas-P. Gérard-F. Golse [5], G. Allaire-G. Bal [1], G. Bal [4], T. Goudon-F. Poupaud [29], T. Goudon-A. Mellet [28].

However, it is a well-known fact that different (formal) methods of approximation can give rise to difference on the limit coefficients, see [31] or [37], [1], [13] for some examples. Furthermore, when starting from multigroup equations and performing the diffusion approximation limit, one usually obtains a single diffusion equation in the space variable only, see [2], [13]: the limit procedure forgets the multigroup aspect. This is because the system is considered to relax towards an equilibrium under both elastic and inelastic collisions at the same scale. However, it could be interesting to derive more complex models, which retain the energy as a variable and where the diffusion as well as the inelastic collisions couple the various energy levels. This is the goal of the present paper. To that purpose, our analysis is inspired by reasonings developed in the modeling of semiconductors devices where, instead of obtaining a drift-diffusion equation, we are led to an intermediate system, by keeping the energy as a variable, see Ben Abdallah-P. Degond [9], P. Degond [18, 19]. We also mention the recent application to the phonons dynamics by J. P. Bourgade [11]. These so-called SHE-models have been shown to be very accurate, in particular for numerical simulations. It is worth noticing at the moment that the energy coupling can be obtained either through the zeroth order terms when the inelastic collisions are treated as perturbations, or through the diffusion operator when inelastic processes are treated in a more intricate way, as it has been done by P. Degond [19], in the framework of semiconductors theory, for continuous energy levels. However, the analysis developed in the present paper, though largely inspired from [19], requires less restrictive hypotheses (see in particular Section 3 and Proposition $5)$. Note that this work is only concerned with the diffusion approximation problem; the homogenization question will be addressed elsewhere. Note also that we have choosen to treat the evolution problem but, of course, our analysis can be applied to eigenvalue problems as well.

The paper is organized as follows. In Section 2, we will set up precisely the multigroup aspect on the Boltzmann equation. Section 3 is devoted to a discussion of some properties 
of the collision operator. In particular, we aim at splitting the Boltzmann operator into an elastic part, that leaves the number of neutrons in a given energy level unchanged, and an inelastic part. This can be done either locally or not as far as the energy variable is concerned. In some sense, the latter retains some relevant information on the energy exchange during the collisions with the medium. In Section 4 we formally discuss the small mean free path asymptotic limit with the requirement that elastic, or quasi-elastic, processes dominate. Actually, our method can be viewed as a heuristic procedure to derive macroscopic models, which are intermediate between a full kinetic description and a simple diffusion equation. Finally, Section 5 is concerned with a rigorous proof of convergence.

\section{Multigroup equations}

The evolution of the population of neutrons is described through an equation relating transport to interaction processes as follows

$$
\left\{\begin{array}{l}
\left(\partial_{t}+v \cdot \nabla_{x}\right) f=Q(f), \\
Q(f)=\frac{1}{\left|S^{N-1}\right|} \int_{\mathbb{R}^{N}} \sigma\left(x, v, v^{\prime}\right) f\left(v^{\prime}\right) d v^{\prime}-\Sigma(x, v) f(v), \\
\Sigma(x, v)=\frac{1}{\left|S^{N-1}\right|} \int_{\mathbb{R}^{N}} \sigma\left(x, v^{\prime}, v\right) d v^{\prime}
\end{array}\right.
$$

The third relation means that the operator $Q$ is conservative, or critical, in the sense that, neglecting integrability questions for the time being, we have

$$
\int_{\mathbb{R}^{N}} Q(f) d v=0
$$

Compared to the Introduction, we have changed the cross-sections by introducing the normalization by $\frac{1}{S^{N-1}}$ for pure convenience; it will allow us to work with normalized measure and will simplify some forthcoming computations.

We write the velocity of the particles as follows

$$
\left\{\begin{array}{l}
v=|v| \omega=\sqrt{2 e / m} \omega, \\
\omega=v /|v|=\text { angular variable } \in S^{N-1}, \\
e=m v^{2} / 2=\text { energy variable } \in \mathbb{R}^{+}
\end{array}\right.
$$

Denoting by $d \omega$ the normalized Euclidian measure on $S^{N-1}$, we have $d v=\left|S^{N-1}\right| r^{N-1} d r d \omega$, with $r=|v|=\sqrt{2 e / m}$, and therefore $d r=\frac{d e}{\sqrt{2 m e}}$. We deduce the following change of variable formula

$$
\int_{\mathbb{R}^{N}} \varphi(v) d v=\int_{0}^{\infty}\left|S^{N-1}\right| \int_{S^{N-1}} \varphi(\sqrt{2 e / m} \omega)(2 e / m)^{N / 2} \frac{d e}{2 e} d \omega
$$

which applies to any integrable function $\varphi$. 
Let us discretize the energy levels by introducing the energy step $\varepsilon>0$ (we point out that $\varepsilon$ will remain fixed throughout the paper, and will not tend to zero). We set $E_{i}=[i \varepsilon,(i+1) \varepsilon[$ and then, we consider

$$
f_{i}(t, x, \omega)=\frac{1}{2 \varepsilon} \int_{E_{i}} f(t, x, \sqrt{2 e / m} \omega)(2 e / m)^{N / 2} \frac{d e}{2 e}
$$

which will be a new unknown. Equations for the $f_{i}$ 's are obtained by averaging (5). In the left hand side, we approach $v$ by $v_{i}=\sqrt{2 i \varepsilon / m} \omega$ and the transport term is approximately $\left(\partial_{t}+v_{i} \cdot \nabla_{x}\right) f_{i}$. For the collision term, one has

$$
\begin{aligned}
& \frac{1}{2 \varepsilon} \int_{E_{i}} Q(f)(t, x,|v| \omega)|v|^{N} \frac{d e}{2 e} \\
& =\frac{1}{2 \varepsilon} \int_{E_{i}}\left(\int_{0}^{\infty} \int_{S^{N-1}} \sigma\left(\sqrt{2 e / m} \omega, \sqrt{2 e^{\prime} / m} \omega^{\prime}\right) f\left(\sqrt{2 e^{\prime} / m} \omega^{\prime}\right)\left(2 e^{\prime} / m\right)^{N / 2} \frac{d e^{\prime}}{2 e^{\prime}} d \omega^{\prime}\right. \\
& \left.-\int_{0}^{\infty} \int_{S^{N-1}}^{\sigma} \sigma\left(\sqrt{2 e^{\prime} / m} \omega^{\prime}, \sqrt{2 e / m} \omega\right)\left(2 e^{\prime} / m\right)^{N / 2} \frac{d e^{\prime}}{2 e^{\prime}} d \omega^{\prime} f(\sqrt{2 e / m} \omega)\right)(2 e / m)^{N / 2} \frac{d e}{2 e} \\
& =\frac{1}{2 \varepsilon} \sum_{j} \int_{E_{j}} \int_{S^{N-1}}\left(\int_{E_{i}} \sigma\left(\sqrt{2 e / m} \omega, \sqrt{2 e^{\prime} / m} \omega^{\prime}\right)(2 e / m)^{N / 2} \frac{d e}{2 e}\right) \\
& -\frac{1}{2 \varepsilon} \int_{E_{i}} f\left(\sqrt{2 e^{\prime} / m} \omega^{\prime}\right)\left(2 e^{\prime} / m\right)^{N / 2} \frac{d e^{\prime}}{2 e^{\prime}} d \omega^{\prime} \\
& \quad\left(\sum_{j} \int_{E_{j}} \int_{S^{N-1}} \sigma\left(\sqrt{2 e^{\prime} / m} \omega^{\prime}, \sqrt{2 e / m} \omega\right)\left(2 e^{\prime} / m\right)^{N / 2} \frac{d e^{\prime}}{2 e^{\prime}} d \omega^{\prime}\right)(2 e / m)^{N / 2} \frac{d e}{2 e}
\end{aligned}
$$

Then, we suppose that $\sigma$ does not vary too much as the energy variables $\left(e, e^{\prime}\right)$ belong to the set $E_{i} \times E_{j}$ and we make the following approximation:

$$
\text { For } e^{\prime} \in E_{j}, \quad \sigma_{i j}\left(\omega, \omega^{\prime}\right) \sim \int_{E_{i}} \sigma\left(\sqrt{2 e / m} \omega, \sqrt{2 e^{\prime} / m} \omega^{\prime}\right)(2 e / m)^{N / 2} \frac{d e}{2 e},
$$

so that $Q(f)$ is replaced by

$$
Q(f)_{i}(\omega)=\sum_{j} \int_{S^{N-1}} \sigma_{i j}\left(\omega, \omega^{\prime}\right) f_{j}\left(\omega^{\prime}\right) d \omega^{\prime}-\Sigma_{i}(\omega) f_{i}(\omega)
$$

where

$$
\Sigma_{i}(\omega)=\sum_{j} \int_{S^{N-1}} \sigma_{j i}\left(\omega^{\prime}, \omega\right) d \omega^{\prime} .
$$

These notations lead to the following multigroup kinetic equation

$$
\left(\partial_{t}+v_{i} \cdot \nabla_{x}\right) f_{i}=Q(f)_{i} .
$$


REMARK 1 Of course, the subdomains $E_{i}$ can be defined by using another discretization rule. For instance, one often discretizes the energy range by means of the lethargy $\lambda(e)=$ $\ln \left(e_{r e f} / e\right)$ and $E_{i}=\left\{e \geq 0, \lambda(e) \in\left[\lambda_{i}, \lambda_{i+1}[\}\right.\right.$, see [41].

\section{Splitting of the collision operator}

In this section, we introduce two different splittings of the collision operator into elastic and inelastic parts. Here and below "elastic" means that the operator leaves invariant the total number of neutrons on a given energy level. As in [19], we will see that it is relevant to use a convex combination of these splittings. For convenience, we skip the space dependence, having in mind that the estimates discussed below are uniform with respect to $x$. Besides, we shall only state precisely the assumptions on the cross-sections $\sigma_{i j}$ and the properties of the collision operators that will be necessary later on; proofs are postponed into the appendix.

\subsection{First splitting}

Let us split the collision operator (6) as follows

$$
\begin{gathered}
Q(f)_{i}(\omega)=\sum_{j} \int_{S^{N-1}}\left(\sigma_{i j}\left(\omega, \omega^{\prime}\right) f_{j}\left(\omega^{\prime}\right)-\sigma_{j i}\left(\omega^{\prime}, \omega\right) f_{i}\left(\omega^{\prime}\right)\right) d \omega^{\prime} \\
+\int_{S^{N-1}}\left(\sum_{j} \sigma_{j i}\left(\omega^{\prime}, \omega\right)\right)\left(f_{i}\left(\omega^{\prime}\right)-f_{i}(\omega)\right) d \omega^{\prime}
\end{gathered}
$$

The first operator modifies energy while angle remains unchanged, thus, we refer to it as the inelastic part, denoted by $Q_{0}^{\text {inel}}$; the second operator modifies the velocity direction while energy is conserved, thus, we called it the elastic part, denoted by $Q_{0}^{e l}$. Let us set

$$
\Gamma_{i}\left(\omega^{\prime}, \omega\right)=\sum_{j} \sigma_{j i}\left(\omega^{\prime}, \omega\right), \quad \Sigma_{i}(\omega)=\int_{S^{N-1}} \Gamma_{i}\left(\omega^{\prime}, \omega\right) d \omega^{\prime}
$$

then we have

$$
Q_{0}^{e l}(f)_{i}=\int_{S^{N-1}} \Gamma_{i}\left(\omega^{\prime}, \omega\right)\left(f_{i}\left(\omega^{\prime}\right)-f_{i}(\omega)\right) d \omega^{\prime}=K(f)_{i}-\Sigma_{i} f_{i}
$$

Let us now state the first assumptions concerning the kernel $\sigma_{i j}\left(\omega, \omega^{\prime}\right)$ :

$$
\text { First symmetry assumption: } \Sigma_{i}(\omega)=\int_{S^{N-1}} \Gamma_{i}\left(\omega^{\prime}, \omega\right) d \omega^{\prime}=\int_{S^{N-1}} \Gamma_{i}\left(\omega, \omega^{\prime}\right) d \omega^{\prime} .
$$

In order to derive the coercivity properties of the operator $Q_{0}^{e l}$, we also require the following hypothesis:

$$
\left\{\begin{array}{c}
\text { There exists a sequence of positive reals }\left(\gamma_{i}\right)_{i \in \mathbb{N}} \\
\text { and a constant } M_{0} \text { such that } \gamma_{i} \leq \Gamma_{i}\left(\omega^{\prime}, \omega\right) \leq M_{0} \gamma_{i}
\end{array}\right.
$$


For technical purposes now, we introduce a sequence $\left(B_{i}\right)_{i \in \mathbb{N}}$ of positive numbers, satisfying:

(h3) Weight assumption: $\left(B_{i}\right)_{i \in \mathbb{N}}$ is such that $\sum_{i} B_{i} \gamma_{i}=M_{1}<\infty$,

(it is easy to check that such a sequence always exists). Note that $(h 2)$ and $(h 3)$ yield

$$
\sum_{i} B_{i} \int_{S^{N-1}} \Sigma_{i}(\omega) d \omega \leq M_{0} M_{1}
$$

It is worth having in mind the following simple example of the isotropic Boltzmann equation, with typically $B_{i}=e^{-i}$,

$$
\sigma_{i j}\left(\omega, \omega^{\prime}\right)=c_{i} c_{j} B_{i}, \quad c_{i} B_{i} \in \ell^{1} .
$$

This leads to $\Gamma_{i}\left(\omega, \omega^{\prime}\right)=\|c B\|_{\ell^{1}} c_{i}$ and (h2) is fulfilled with $M_{0}=\|c B\|_{\ell^{1}}^{2}$, and $\gamma_{i}=c_{i}$.

We now introduce the following functional spaces

$$
\begin{aligned}
& \mathbb{E}=\left\{f: \mathbb{N} \times S^{N-1} \rightarrow \mathbb{R} \text { such that }\|f\|_{\mathbb{E}}^{2}=\sum_{i} \int_{S^{N-1}}\left|f_{i}(\omega)\right|^{2} \frac{\Sigma_{i}(\omega)}{B_{i}} d \omega<\infty\right\}, \\
& \mathbb{F}=\left\{f: \mathbb{N} \times S^{N-1} \rightarrow \mathbb{R} \text { such that }\|f\|_{\mathbb{F}}^{2}=\sum_{i} \int_{S^{N-1}}\left|f_{i}(\omega)\right|^{2} \frac{1}{\Sigma_{i}(\omega) B_{i}} d \omega<\infty\right\} .
\end{aligned}
$$

We shall identify the space

$$
\mathbb{L}=\left\{f: \mathbb{N} \times S^{N-1} \rightarrow \mathbb{R} \text { such that }\|f\|_{\mathbb{L}}^{2}=\sum_{i} \int_{S^{N-1}}\left|f_{i}(\omega)\right|^{2} \frac{1}{B_{i}} d \omega<\infty\right\}
$$

with its dual when equipped with the inner product

$$
(f, g)_{\mathbb{L}}=\sum_{i} \int_{S^{N-1}} f_{i}(\omega) g_{i}(\omega) \frac{1}{B_{i}} d \omega
$$

Consequently, we note that

$$
\left|\sum_{i} \int_{S^{N-1}} f_{i}(\omega) g_{i}(\omega) \frac{1}{B_{i}} d \omega\right| \leq\|f\|_{\mathbb{E}}\|g\|_{\mathbb{F}}
$$

for any $f \in \mathbb{E}, g \in \mathbb{F}$ and we can identify $\mathbb{F}$ with the dual $\mathbb{E}^{\prime}$. We are now ready to establish the main properties of the elastic operator $Q_{0}^{e l}$.

Proposition 1 Assume that (h1) holds. Then, $Q_{0}^{e l} \in \mathcal{L}(\mathbb{E}, \mathbb{F})$ with $\left\|Q_{0}^{e l}(f)\right\|_{\mathbb{F}} \leq 2\|f\|_{E}$,

i) Conservation property: $\int_{S^{N-1}} Q_{0}^{e l}(f)_{i} d \omega=0$ (at least formally; see Remark 3),

$\mathrm{RR} \mathrm{n}^{\circ} 4302$ 
ii) Dissipativity property: for all $f, g$ in $\mathbb{E}$, we set $\mathcal{B}_{0}(f, g)=-\sum_{i} \int_{S_{N-1}} Q_{0}^{e l}(f)_{i} g_{i} B_{i}^{-1} d \omega$. Then, $\mathcal{B}_{0}$ is bilinear continuous on $\mathbb{E}$ and satisfies

$$
\begin{aligned}
\mathcal{B}_{0}(f, f) & =1 / 2 \sum_{i} \int_{S^{N-1}} \int_{S^{N-1}} \Gamma_{i}\left(\omega^{\prime}, \omega\right)\left|f_{i}(\omega)-f_{i}\left(\omega^{\prime}\right)\right|^{2} B_{i}^{-1} d \omega^{\prime} d \omega \\
& \geq 1 / 2\left\|Q_{0}^{e l}(f)\right\|_{\mathbb{F}}^{2} .
\end{aligned}
$$

iii) The eigenspace $\operatorname{Ker}\left(Q_{0}^{e l}\right)$ is the space $\mathcal{E}_{0}$ of functions in $\mathbb{E}$ which do not depend on the angular variable.

REMARK 2 Under Hypothesis (h2), one remarks that $\mathcal{E}_{0}=\left\{f: \mathbb{N} \rightarrow \mathbb{R}, \sum_{i} f_{i}^{2} \gamma_{i} B_{i}^{-1}<\infty\right\}$ does not reduce to $\{0\}$. For instance, with (h2)-(h3) it contains $\left\{f_{i}=B_{i} g_{i}, g \in \ell^{\infty}\right\} \subset \mathcal{E}_{0}$. Indeed, for such a $f$, we get

$$
0 \leq \sum_{i} \int_{S^{N-1}} \Sigma_{i} f_{i}^{2} / B_{i} d \omega=\sum_{i}\left(g_{i}^{2} B_{i} \int_{S^{N-1}} \Sigma_{i} d \omega\right) \leq M_{1} M_{0}\|g\|_{\ell^{\infty}}^{2}<\infty .
$$

When taking into account time and space variable we will work with sequences of functions $f_{i}(t, x)$ that satisfy the corresponding integrability condition.

REMARK 3 Assumption (h3) also implies that $\mathbb{F}$ is a subset of integrable functions, for the measure $d \omega \otimes d i$, where di is the counting measure on $\mathbb{N}$, since one has

$$
\sum_{i} \int_{S^{N-1}}\left|g_{i}\right| d \omega \leq\left(\sum_{i} \int_{S^{N-1}} g_{i}^{2} /\left(B_{i} \Sigma_{i}\right) d \omega\right)^{1 / 2}\left(\sum_{i} \int_{S^{N-1}} B_{i} \Sigma_{i} d \omega\right)^{1 / 2} \leq\|g\|_{\mathbb{F}}\left(M_{0} M_{1}\right)^{1 / 2} .
$$

$A$ similar conclusion holds for $f \in \mathbb{E}$ if one assumes

$$
\sum_{i} \int_{S^{N-1}} B_{i} / \Sigma_{i} d \omega<\infty
$$

For (9), this means that $B / c \in \ell^{1}$.

In order to state a coercivity property in a useful setting, we introduce the following norm

$$
N(f)=\sum_{i} \int_{S^{N-1}}\left|f_{i}(\omega)\right|^{2} \frac{\gamma_{i}}{B_{i}} d \omega .
$$

In view of Hypothesis (h2), it is readily seen that $N(f)$ defines an equivalent norm on $\mathbb{E}$. Actually we have

$$
N(f)^{2} \leq\|f\|_{\mathbb{E}}^{2} \leq M_{0} N(f)^{2} .
$$


Corollary 1 Under Hypotheses (h1-h3), the following coercivity estimate

$$
\mathcal{B}_{0}(f, f) \geq N^{2}(f-\langle f\rangle)
$$

holds for any $f \in \mathbb{E}$, where one denotes $\langle f\rangle_{i}=\int_{S^{N-1}} f_{i}(\omega) d \omega$.

REMARK 4 The adjoint operator of $Q_{0}^{e l}$ reads

$$
\begin{aligned}
Q_{0}^{e l, *}(g)_{i} & =\int_{S^{N-1}} \Gamma_{i}\left(\omega, \omega^{\prime}\right) g_{i}\left(\omega^{\prime}\right) d \omega^{\prime}-\Sigma_{i}(\omega) g_{i}(\omega) \\
& =\int_{S^{N-1}} \Gamma_{i}\left(\omega, \omega^{\prime}\right)\left(g_{i}\left(\omega^{\prime}\right)-g_{i}(\omega)\right) d \omega
\end{aligned}
$$

\section{$3.2 \quad$ Second splitting}

On the other hand, we can also introduce the following splitting into elastic and inelastic operators

$$
Q(f)_{i}=Q_{1}^{e l}(f)_{i}+Q_{1}^{i n e l}(f)_{i}
$$

with

$$
\left\{\begin{array}{l}
Q_{1}^{e l}(f)_{i}=\sum_{j} \int_{S^{N-1}} \sigma_{i j}\left(\omega, \omega^{\prime}\right)\left(f_{j}\left(\omega^{\prime}\right)-f_{j}(\omega)\right) d \omega^{\prime} \\
Q_{1}^{i n e l}(f)_{i}=\sum_{j}\left(\int_{S^{N-1}} \sigma_{i j}\left(\omega, \omega^{\prime}\right) d \omega^{\prime} f_{j}(\omega)\right)-f_{i}(\omega) \sum_{j} \int_{S^{N-1}} \sigma_{j i}\left(\omega^{\prime}, \omega\right) d \omega^{\prime}
\end{array}\right.
$$

The boundedness of $Q_{1}^{e l}$ relies on the following assumption

$$
\left\{\begin{array}{l}
\text { There exists a constant } M_{2} \text { such that } \\
\sum_{j} \int_{S^{N-1}} \sigma_{i j}\left(\omega, \omega^{\prime}\right) B_{j} d \omega^{\prime} \leq M_{2} \gamma_{i} B_{i}
\end{array}\right.
$$

It is worth pointing out that this assumption is a straightforward consequence of (h2-h3), if we assume the following relation, known as the detailed balance principle

$$
\sigma_{i j}\left(\omega, \omega^{\prime}\right) B_{j}=\sigma_{j i}\left(\omega^{\prime}, \omega\right) B_{i} .
$$

We also need the

$\left(h 1^{\prime}\right) \quad$ Second symmetry condition: $\int_{S^{N-1}} \sigma_{i j}\left(\omega^{\prime}, \omega\right) d \omega^{\prime}=\int_{S^{N-1}} \sigma_{i j}\left(\omega, \omega^{\prime}\right) d \omega^{\prime}$.

Remark that (h1') is stronger than (h1), and that (h4) as well as (h2) are fulfilled if we assume $\sigma_{i j} \leq M \gamma_{i} B_{i} \gamma_{j}$.

$\mathrm{RR} \mathrm{n}^{\circ} 4302$ 
Proposition 2 Assume that ( $h 1^{\prime}$, h2, h3, h4) hold. Then, $Q_{1}^{e l} \in \mathcal{L}(\mathbb{E}, \mathbb{F})$ with $\left\|Q_{1}^{e l}(f)\right\|_{\mathbb{F}} \leq$ $2 M_{2}^{1 / 2}\|f\|_{\mathbb{E}}$, and we have

i) Conservation property: $\int_{S^{N-1}} Q_{1}^{e l}(f)_{i} d \omega=0$,

ii) For all $f, g$ we set $\mathcal{B}_{1}(f, g)=-\sum_{i} \int_{S^{N-1}} Q_{1}^{e l}(f)_{i} g_{i} B_{i}^{-1} d \omega$. Then, $\mathcal{B}_{1}$ is bilinear continuous on $\mathbb{E}$ and it satisfies

$\mathcal{B}_{1}(f, g)=\frac{1}{2} \sum_{i, j} \int_{S^{N-1}} \int_{S^{N-1}}\left(f_{j}\left(\omega^{\prime}\right)-f_{j}(\omega)\right)\left(\sigma_{i j}\left(\omega^{\prime}, \omega\right) g_{i}\left(\omega^{\prime}\right)-\sigma_{i j}\left(\omega, \omega^{\prime}\right) g_{i}(\omega)\right) B_{i}^{-1} d \omega^{\prime} d \omega$.

iii) $\mathcal{E}_{0} \subset \operatorname{Ker}\left(Q_{1}^{e l}\right)$, and the following estimate holds

$$
\left|\mathcal{B}_{1}(f, f)\right| \leq M_{0} \sqrt{2 M_{2}} N(f-\langle f\rangle)^{2} .
$$

Of course functions depending only on the energy variable belongs to $\operatorname{Ker}\left(Q_{1}^{e l}\right)$; however, the kernel of $Q_{1}^{e l}$ contains much more functions. Note that iii) is slightly sharper than the estimate obtained by using the norm of $Q_{1}^{e l}$; it can be improved under more restrictive assumptions on the kernels.

Corollary 2 Under the

$\left(h 1^{\prime \prime}\right) \quad$ Strong symmetry assumption: $\sigma_{i j}\left(\omega, \omega^{\prime}\right)=\sigma_{i j}\left(\omega^{\prime}, \omega\right)$

and

$$
\left(h 2^{\prime \prime}\right) \quad \sigma_{i j}\left(\omega, \omega^{\prime}\right) \leq M \gamma_{i} B_{i} \gamma_{j} \quad \text { with } \sum_{i} B_{i} \gamma_{i}=M_{1}<\infty,
$$

the operator $Q_{0}^{e l}$ is self-adjoint and, concerning $Q_{1}^{e l}$, we have

$$
\mathcal{B}_{1}(f, g)=1 / 2 \sum_{i, j} \int_{S^{N-1}} \int_{S^{N-1}} \sigma_{i j}\left(\omega, \omega^{\prime}\right)\left(f_{j}\left(\omega^{\prime}\right)-f_{j}(\omega)\right)\left(g_{i}\left(\omega^{\prime}\right)-g_{i}(\omega)\right) B_{i}^{-1} d \omega^{\prime} d \omega .
$$

As a consequence, if $\langle f\rangle=0$, then iii) becomes

$$
\left|\mathcal{B}_{1}(f, f)\right| \leq M M_{1} N(f)^{2} .
$$

REMARK 5 Notice that (h1") implies (h1') while (h2") implies both (h2) and (h3) with $M_{0}=$ $M M_{1}$, and (h4) with $M_{2}=M_{1}$. The bound from below in (h2) is fulfilled if one assumes a similar estimate from below on the $\sigma_{i j}$ 's.

REMARK 6 The previous symmetry conditions are included in

$$
\sigma_{i j}\left(\omega, \omega^{\prime}\right) B_{j}=\sigma_{i j}\left(\omega^{\prime}, \omega\right) B_{j}=\sigma_{j i}\left(\omega^{\prime}, \omega\right) B_{i}=\sigma_{j i}\left(\omega, \omega^{\prime}\right) B_{i}
$$

which also implies that $Q_{1}^{e l}$ is self-adjoint. This corresponds to the symmetry assumption used in [19]; but here we aim at dealing with a larger class of collision kernels.

INRIA 
Let us go back to the fundamental example (9). Denote $C=\sum_{i} c_{i} B_{i}<\infty$. One has in this simple case

$$
\left\{\begin{array}{l}
Q_{0}^{e l}(f)_{i}=C c_{i}\left(\left\langle f_{i}\right\rangle-f_{i}\right), \\
Q_{1}^{e l}(f)_{i}=c_{i} B_{i} \sum_{j} c_{j}\left(\left\langle f_{j}\right\rangle-f_{j}\right) .
\end{array}\right.
$$

Therefore, we get

$$
\left\{\begin{array}{l}
\mathcal{B}_{0}(f, g)=\sum_{i, j} c_{i} B_{i}^{-1} c_{j} B_{j}\left(\left\langle f_{i}\right\rangle\left\langle g_{i}\right\rangle-\left\langle f_{i} g_{i}\right\rangle\right) \\
\mathcal{B}_{1}(f, g)=\sum_{i, j} c_{i} c_{j}\left(\left\langle f_{j}\right\rangle\left\langle g_{i}\right\rangle-\left\langle f_{j} g_{i}\right\rangle\right) .
\end{array}\right.
$$

We write $g_{i}=\left\langle g_{i}\right\rangle+r_{i}$ where $\left\langle r_{i}\right\rangle=0$. It yields

$$
\begin{aligned}
\mathcal{B}_{1}(f, g) & =\sum_{i, j} c_{i} c_{j}\left(\left\langle f_{j}\right\rangle\left\langle g_{i}\right\rangle-\left\langle f_{j}\left\langle g_{i}\right\rangle\right\rangle-\left\langle f_{j} r_{i}\right\rangle\right) \\
& =-\sum_{i, j} c_{i} c_{j}\left\langle f_{j} r_{i}\right\rangle=-\sum_{i, j} c_{i} c_{j} \int_{S^{N-1}} f_{j} r_{i} d \omega \\
& =-\int_{S^{N-1}}\left(\sum_{j} c_{j} f_{j}\right)\left(\sum_{i} c_{i} r_{i}\right) d \omega .
\end{aligned}
$$

If $g$ lies in the orthogonal set of $\operatorname{Ran}\left(Q_{1}^{e l}\right)$, we deduce that $\sum_{i} c_{i} r_{i}(\omega)$ vanishes for almost all $\omega \in S^{N-1}$.

REMARK 7 The adjoint operator of $Q_{1}^{e l}$ is given by:

$$
Q_{1}^{e l, *}(g)_{i}=\sum_{j} \int_{S^{N-1}} \sigma_{j i}\left(\omega^{\prime}, \omega\right)\left(g_{j}\left(\omega^{\prime}\right)-g_{j}(\omega)\right) \frac{B_{i}}{B_{j}} d \omega^{\prime} .
$$

In particular, we note that, if the relation (11) holds, then $Q_{1}^{e l}$ is a self-adjoint operator.

\subsection{Combination of the splitting}

From now on, we assume that (h1'), (h2), (h3), (h4) hold and we denote by (H) this set of hypotheses. The idea will be to combine $Q_{1}^{e l}$ with $Q_{0}^{e l}$ so that the coercivity of the latter compensates the lack of positivity of the former; we are thus able to preserve the crucial dissipation properties. Let us consider the following elastic operator, obtained as a convex combination of $Q_{0}^{e l}$ and $Q_{1}^{e l}$; for $\theta \in[0,1]$, we set

$$
Q_{\theta}=\theta Q_{1}^{e l}+(1-\theta) Q_{0}^{e l} .
$$

One deduces from Corollary 1 and Proposition 2 that

$$
\mathcal{B}_{\theta}(f, f)=-\sum_{i} \int_{S^{N-1}} Q_{\theta}(f)_{i} f_{i} B_{i}^{-1} d \omega \geq\left(1-\theta\left(1+M_{0} \sqrt{2 M_{2}}\right)\right) N(f-\langle f\rangle)^{2}
$$

holds for any $f \in \mathbb{E}$. We are thus led to the following statement.

$\mathrm{RR} \mathrm{n}^{\circ} 4302$ 
Proposition 3 Let $\theta \in\left[0,\left(1+M_{0} \sqrt{2 M_{2}}\right)^{-1}\right.$ [. Then, there exists a constant $\kappa_{\theta}>0$ such that, for any $f \in \mathbb{E}$, we have

$$
\mathcal{B}_{\theta}(f, f) \geq \kappa_{\theta} N(f-\langle f\rangle)^{2} .
$$

Consequently, the set of equilibria $\mathcal{E}_{\theta}=\operatorname{Ker}\left(Q_{\theta}\right)$ coincides with $\mathcal{E}_{0}$ (the set of functions depending only on the energy level). If one assumes (h1") and (h2"), the domain for $\theta$ enlarges to $\left[0,\left(1+M_{0}\right)^{-1}[\right.$.

One may also establish the following Fredholm alternative.

Proposition 4 For any $h \in \mathbb{F}$ the problem to find $f \in \mathbb{E}$ such that $Q_{\theta}(f)=h$ has a solution if and only if $\langle h\rangle=0$. The solution is unique in $\mathbb{E}_{0}=\{f \in \mathbb{E}$ such that $\langle f\rangle=0\}$ and satisfies

$$
N(f) \leq\left(1 / \sqrt{\kappa_{\theta}}\right)\|h\|_{\mathbb{F}} .
$$

Proof. Since the operator $Q_{\theta}$ is clearly conservative, the condition of null average on the data $h$ is necessary. The problem recasts into the following variational formulation

$$
\forall \varphi \in \mathbb{E}_{0}, \quad \mathcal{B}_{\theta}(f, \varphi)=\sum_{i} \int_{S^{N-1}} h_{i} \varphi_{i} B_{i}^{-1} d \omega=(h, \varphi)_{\mathbb{L}}
$$

and we conclude by applying the Lax-Milgram theorem.

Of course, one has a similar statement for the adjoint operator: $Q_{\theta}^{*}$ is defined by

$$
Q_{\theta}^{*}=\theta Q_{1}^{e l, *}+(1-\theta) Q_{0}^{e l, *},
$$

and fulfills the same coercivity property as $Q_{\theta}$ with the same constant of coercivity, since

$$
\mathcal{B}_{\theta}(f, f)=-\sum_{i} \int_{S^{N-1}} Q_{\theta}^{*}(f)_{i} f_{i} B_{i}^{-1} d \omega .
$$

Therefore we have

Corollary 3 For any $h \in \mathbb{F}$ there exists $f \in \mathbb{E}$ such that $Q_{\theta}^{*}(f)=h$ if and only if $\langle h\rangle=0$. Moreover, there exists a unique such function in $\mathbb{E}_{0}=\{f \in \mathbb{E}$ such that $\langle f\rangle=0\}$, and this solution satisfies

$$
N(f) \leq\left(1 / \sqrt{\kappa_{\theta}}\right)\|h\|_{\mathbb{F}}
$$

\section{Formal approach}

The starting point of the asymptotic study is the following rescaled equation

$$
\partial_{t} f_{i}^{\eta}+\frac{1}{\eta} v_{i} \cdot \nabla_{x} f_{i}^{\eta}=\frac{1}{\eta^{2}}\left(Q^{\eta}\left(f^{\eta}\right)\right)_{i} .
$$

INRIA 
Precisely, we consider the situation where the mean free path $\eta>0$ is small at time scale of order $1 / \eta$. On the other hand, we assume that the collision operator $Q^{\eta}$ splits as follows

$$
Q^{\eta}(f)=Q_{\theta}(f)+\eta^{2} Q_{\theta}^{\text {inel }}(f),
$$

which means that inelastic collisions are of order $\eta^{2}$ compared to the elastic ones. This agrees with the fact that dominant scattering events are elastic. Of course, in this splitting $\theta$ is fixed and cannot be too small compared to $\eta$. In particular, it is also required that $\theta$ belongs to the range which guarantees the coercivity of the operator $Q_{\theta}$, see Proposition 3. Our aim is to describe the asymptotic behaviour of the solutions of (13) as $\eta \rightarrow 0$. We will obtain a set of diffusion equations, which can be viewed as a semi-discrete (with respect to the energy) version of the SHE-system of [19], for the limit macroscopic density with a coupling of the energy levels due to the action of the operator $Q_{1}^{e l}$ and/or the inelastic terms.

\subsection{Formal Hilbert expansion}

We can guess the limit behaviour by inserting the formal ansatz

$$
f^{\eta}=f^{0}+\eta f^{1}+\eta^{2} f^{2}+\ldots
$$

into (13) and then, we identify the terms that arise with the same power of $\eta$. As usual, the $\eta^{-2}$ terms lead to

$$
Q_{\theta}\left(f^{0}\right)=0
$$

which means, by Proposition 3, that $\left(f^{0}\right)_{i}(\omega)=\rho_{i} \in \mathcal{E}_{0}$ does not depend on the angular variable. Hence, one expects that the asymptotic limit is entirely determined in terms of "macroscopic" quantities. Next, the $\eta^{-1}$ equation reads

$$
Q_{\theta}\left(f^{1}\right)=v \cdot \nabla_{x} \rho,
$$

while the $\eta^{0}$ equation is

$$
Q_{\theta}\left(f^{2}\right)+Q_{\theta}^{i n e l}(\rho)=v \cdot \nabla_{x} f^{1}+\partial_{t} \rho .
$$

Integrating with respect to $\omega$, we are led to the following relation

$$
\partial_{t} \rho_{i}+\operatorname{div}_{x}\left(\int_{S^{N-1}} \sqrt{2 e_{i} / m} \omega f_{i}^{1}(\omega) d \omega\right)=\int_{S^{N-1}} Q_{\theta}^{i n e l}(\rho) d \omega .
$$

The usual strategy consists in inverting the $\eta^{-1}$ equation; one expects in this way to determine the current $\int_{S^{N-1}} \sqrt{2 e_{i} / m} \omega f_{i}^{1}(\omega) d \omega$ in the previous equation as a linear function of $\nabla_{x} \rho$, i.e. a Fick's relation. The difficulty of this method here comes from the action of the operator $Q_{1}^{e l}$ which mixes the energy levels. In particular, if $\rho_{i}$ depends only on the energy and $f_{i}(\omega)$ depends on the two variables, in general one has

$$
\begin{aligned}
Q_{1}(\rho f)_{i} & =\sum_{j} \int_{S^{N-1}} \sigma_{i j}\left(\omega, \omega^{\prime}\right) \rho_{j}\left(f_{j}\left(\omega^{\prime}\right)-f_{j}(\omega)\right) d \omega^{\prime} \\
& \neq \rho_{i} Q_{1}(f)_{i}=\rho_{i} \sum_{j} \int_{S^{N-1}} \sigma_{i j}\left(\omega, \omega^{\prime}\right)\left(f_{j}\left(\omega^{\prime}\right)-f_{j}(\omega)\right) d \omega^{\prime}
\end{aligned}
$$

$\mathrm{RR} \mathrm{n}^{\circ} 4302$ 
(while $\left.Q_{0}(\rho f)_{i}=\rho_{i} Q_{0}(f)_{i}\right)$. However, we can solve the problem as follows. Let us define $\bar{\chi}^{i} \in\left(\mathbb{E}_{0}\right)^{N}$ solution of

$$
Q_{\theta}\left(\bar{\chi}^{i}\right)_{i_{0}}=-v_{i_{0}} \delta_{i_{0} i}
$$

where $\delta_{i_{0} i}=1$ if $i=i_{0}$, and 0 if $i \neq i_{0}$. The equation has to be understood componentwise, and this definition makes sense thanks to Proposition 4 (since $\int_{S^{N-1}} \omega d \omega=0$ ). Then one checks that

$$
f_{i_{0}}^{1}=\sum_{i} \bar{\chi}_{i_{0}}^{i} \cdot \nabla_{x} \rho_{i}
$$

provides a solution to the $\eta^{-1}$ equation. Plugging this expression into (14) gives

$$
\left\{\begin{array}{l}
\partial_{t} \rho_{i_{0}}-\nabla_{x}\left(\sum_{i} D_{i_{0} i} \nabla_{x} \rho_{i}\right)=q(\rho)_{i_{0}}, \\
q(\rho)_{i_{0}}=\int_{S^{N-1}}\left[Q_{\theta}^{i n e l}(\rho)\right]_{i_{0}} d \omega \\
D_{i_{0} i}=-\int_{S^{N-1}} v_{i_{0}} \otimes \bar{\chi}_{i_{0}}^{i} d \omega
\end{array}\right.
$$

where $\otimes$ stands for the tensor product in $\mathbb{R}^{N}:$ for $\left(a_{1}, \ldots, a_{N}\right)$ and $\left(b_{1}, \ldots, b_{N}\right)$ in $\mathbb{R}^{N}, a \otimes b$ is the $N \times N$ matrix with components $a_{\alpha} b_{\beta}$. We observe in the limit equation a coupling between the energy levels, both from the diffusion matrix and from the right hand side.

A possible strategy to justify these computations would be to start from a solution $\rho$ of the expected limit equation (which has to be studied independently), and to define successively $f^{1}, f^{2}$ in terms of $\rho$ as solutions of the $\eta^{-1}$ and $\eta^{0}$ equations respectively. It remains to estimate the remainder $r^{\eta}=f^{\eta}-\left(\rho+\eta f^{1}+\eta^{2} f^{2}\right)$. The drawback of this method is that it is known to require some regularity on $\rho$, thus on the coefficients. Such an assumption can be unrealistic for neutron transport since the physical properties of the media interacting with the neutrons are usually highly heterogeneous. On the other hand, it could be quite delicate to carry out this strategy in the full generality considered here. Therefore, let us instead develop a duality approach, which will be close to our actual method of proof.

\subsection{Duality interpretation}

Let us assume that $f^{\eta}$ converges to some $f^{0}$ in a suitable sense. Multiplying by $\eta^{2}$ and taking the limit $\eta \rightarrow 0$ in (13), we recover

$$
Q_{\theta}\left(f^{0}\right)_{i}=0, \quad \forall i \in \mathbb{N},
$$

and thus $f_{i}^{0}(t, x, \omega)=\rho_{i}(t, x)$.

We introduce the following macroscopic quantities

$$
\begin{cases}\text { density of } i \text { th energy level } & \rho_{i}^{\eta}(t, x)=\int_{S^{N-1}} f_{i}^{\eta}(t, x, \omega) d \omega, \\ \text { current of } i \text { th energy level } & J_{i}^{\eta}(t, x)=\frac{1}{\eta} \int_{S^{N-1}}\left|v_{i}\right| \omega f_{i}^{\eta}(t, x, \omega) d \omega .\end{cases}
$$


In view of the penalization of the collision term, one expects that $f^{\eta}$ tends to belong to the kernel of $Q_{\theta}$, therefore it is mainly given by its macroscopic part $\rho^{\eta}$, up to a formally small remainder. Hence, let us write a first order expansion of $f^{\eta}$ as follows

$$
f_{i}^{\eta}(t, x, \omega)=\rho_{i}^{\eta}(t, x)+\eta g_{i}^{\eta}(t, x, \omega)
$$

so that $\rho^{\eta} \in \operatorname{Ker}\left(Q_{\theta}\right)$ and

$$
J_{i}^{\eta}(t, x)=\int_{S^{N-1}}\left|v_{i}\right| \omega g_{i}^{\eta}(t, x, \omega) d \omega .
$$

Integrate equation (13) with respect to $\omega$. Since the operator $Q_{\theta}$ has null average on $S^{N-1}$, we get for all $i \in \mathbb{N}$ :

$$
\partial_{t} \rho_{i}^{\eta}+\nabla_{x} \cdot J_{i}^{\eta}=\int_{S^{N-1}} Q_{\theta}^{i n e l}\left(f^{\eta}\right)_{i} d \omega
$$

which is the $\eta$-dependent version of the integrated $\eta^{0}$ equation in the formal expansion above. As $\eta$ goes to 0 , we are formally led to the continuity equation

$$
\partial_{t} \rho_{i}+\nabla_{x} \cdot J_{i}=\int_{S^{N-1}} Q_{\theta}^{i n e l}(\rho)_{i} d \omega .
$$

It remains to find the relation between the limit current $J$ and $\rho$.

Multiply (13) by some $\eta \varphi_{i}(\omega)$. We get

$$
\sum_{i} \frac{1}{\eta} \int_{S^{N-1}} Q_{\theta}\left(f^{\eta}\right)_{i} \varphi_{i} d \omega=\sum_{i} \int_{S^{N-1}}\left(v \cdot \nabla_{x} f^{\eta}\right)_{i} \varphi_{i} d \omega+\eta \sum_{i} \int_{S^{N-1}} \partial_{t} f_{i}^{\eta} \varphi_{i} d \omega
$$

and therefore

$$
\sum_{i} \int_{S^{N-1}} Q_{\theta}\left(g^{\eta}\right)_{i} \varphi_{i} d \omega=\sum_{i}\left(\int_{S^{N-1}}\left|v_{i}\right| \omega \varphi_{i} d \omega\right) \cdot \nabla_{x} \rho_{i}^{\eta}+R^{\eta}
$$

where $R^{\eta}$ is formally of order $\mathcal{O}(\eta)$.

Suppose that, for $i_{0}$ fixed in $\mathbb{N}$, we are able to find the auxiliary function $B_{i} \chi_{i}^{i_{0}}(\omega) \in$ $\left(\mathbb{E}_{0}\right)^{N}$, that solves

$$
Q_{\theta}^{*}\left(B \cdot \chi^{i_{0}}\right)_{i}=v_{i_{0}} B_{i_{0}} \delta_{i_{0} i}=\sqrt{2 e_{i_{0}} / m} B_{i_{0}} \omega \delta_{i_{0} i} .
$$

Since $v_{i_{0}}$ lies in $\mathbb{R}^{N}$, equation (17) holds for the $N$ scalar equations with right hand sides $\omega_{\alpha}$ for $\alpha \in\{1, \ldots, N\}$. Remarking that

$$
\int_{S^{N-1}} \omega d \omega=0
$$

the existence of $\chi^{i_{0}}$ is given by Corollary 3 .

$\mathrm{RR} \mathrm{n}^{\circ} 4302$ 
Hence, for the test function $\varphi_{i}(\omega)$, we choose the components of $\chi_{i}^{i_{0}}(\omega)$ and (16) becomes

$$
\sum_{i} \int_{S^{N-1}} g_{i}^{\eta} Q_{\theta}^{*}\left(B \chi^{i_{0}}\right)_{i} B_{i}^{-1} d \omega=\sum_{i}\left(\int_{S^{N-1}}\left|v_{i}\right| \chi_{i}^{i_{0}} \otimes \omega d \omega\right) \cdot \nabla_{x} \rho_{i}^{\eta}+\mathcal{O}(\eta),
$$

with

$$
\begin{aligned}
\sum_{i} \int_{S^{N-1}} g_{i}^{\eta} Q_{\theta}^{*}\left(B \chi^{i_{0}}\right)_{i} B_{i}^{-1} d \omega & =\sum_{i} \int_{S^{N-1}} g_{i}^{\eta} \sqrt{2 e_{i_{0}} / m} \omega \delta_{i_{0} i} d \omega \\
& =\sqrt{2 e_{i_{0}} / m} \int_{S^{N-1}} \omega g_{i_{0}}^{\eta} d \omega \\
& =J_{i_{0}}^{\eta} .
\end{aligned}
$$

Here, we used the following fact

$$
\sum_{i} \int_{S^{N-1}} Q_{\theta}\left(g^{\eta}\right)_{i} \chi_{i}^{i_{0}} d \omega=\left(Q_{\theta}\left(g^{\eta}\right), B \chi^{i_{0}}\right)_{\mathbb{L}}=\sum_{i} \int_{S^{N-1}} g_{i}^{\eta} Q_{\theta}^{*}\left(B \chi^{i_{0}}\right)_{i} B_{i}^{-1} d \omega .
$$

Thus, passing to the limit $\eta \rightarrow 0$ in (18), we are led to

$$
J_{i_{0}}(t, x)=-\sum_{i} D_{i_{0} i} \nabla_{x} \rho_{i}(t, x)
$$

where the matrix $D$ is defined through the auxiliary function $\chi$ by

$$
D_{i_{0} i}=-\left|v_{i}\right| \int_{S^{N-1}} \chi_{i}^{i_{0}} \otimes \omega d \omega \in \mathcal{M}_{N \times N},
$$

where $\mathcal{M}_{N \times N}$ stands for the space of $N \times N$ matrices.

The formal limit of (13) is therefore the following macroscopic equation

$$
\partial_{t} \rho-\nabla_{x} \cdot\left(D(x) \nabla_{x} \rho\right)=q(\rho),
$$

where the unknown is the sequence $\rho(t, x)=\left\{\rho_{i}(t, x), i \in \mathbb{N}\right\}, D$ is given by (17), (20) and the right hand side by

$$
q(\rho)_{i}=\int_{S^{N-1}} Q_{\theta}^{i n e l}(\rho)_{i} d \omega=\sum_{j} \Lambda_{i j} \rho_{j}-\widetilde{\Sigma}_{i} \rho_{i}
$$

with

$$
\left\{\begin{array}{l}
\Lambda_{i j}=\int_{S^{N-1}} \int_{S^{N-1}} \sigma_{i j}\left(\omega, \omega^{\prime}\right) d \omega d \omega^{\prime}, \\
\widetilde{\Sigma}_{i}=\sum_{j} \int_{S^{N-1}} \int_{S^{N-1}} \sigma_{j i}\left(\omega^{\prime}, \omega\right) d \omega d \omega^{\prime}=\sum_{j} \Lambda_{j i} .
\end{array}\right.
$$

It is also worth splitting the matrix $D$ as follows. The properties discussed on $Q_{0}^{e l}$ allows us to define a unique $\varphi^{i_{0}}(\omega) \in \mathbb{R}^{N}$ verifying

$$
(1-\theta) Q_{0}^{*}\left(B . \varphi^{i_{0}} \delta_{i_{0}} .\right)_{i}=v_{i_{0}} B_{i_{0}} \delta_{i_{0} i}
$$


with $\int_{S^{N-1}} \varphi^{i_{0}}(\omega) d \omega=0$. Then, we split $\chi_{i}^{i_{0}}=\varphi^{i_{0}} \delta_{i_{0} i}+\bar{\chi}_{i}^{i_{0}}$, where

$$
Q_{\theta}^{*}\left(B \cdot \bar{\chi}^{i_{0}}\right)_{i}=-\theta Q_{1}^{*}\left(B \cdot \varphi^{i_{0}} \delta_{i_{0}} \cdot\right)_{i} .
$$

In this way, we can rewrite

$$
\left\{\begin{array}{l}
D_{i_{0} i}=d_{i_{0}} \delta_{i_{0} i}+\Delta_{i_{0} i} \\
d_{i_{0}}=-\left|v_{i_{0}}\right| \int_{S^{N-1}} \varphi^{i_{0}} \otimes \omega d \omega \\
\Delta_{i_{0} i}=-\left|v_{i}\right| \int_{S^{N-1}} \bar{\chi}_{i}^{i_{0}} \otimes \omega d \omega .
\end{array}\right.
$$

Hence, one gets $\left[D \nabla_{x} \rho\right]_{i_{0}}=d_{i_{0}} \nabla_{x} \rho_{i_{0}}+\sum_{i} \Delta_{i_{0}} \nabla_{x} \rho_{i}$. Of course, we can verify readily that this definition of the diffusion matrix coincides with the one obtained in the previous Section.

This limit equation (21) appears as a semi-discrete (in energy) SHE-model with a coupling of the energy levels. Actually, one obtains a hierarchy of possible limit systems:

- A system of uncoupled diffusion equations with respect to the space variable, energy being only a parameter. This arises when inelastic terms are negligeable and $\theta=0$.

- A system of diffusion equations with a coupling of the energy levels through zeroth order terms. This arises with $\theta=0$ and treating inelastic processes as a perturbation. Diffusivity remains locally defined (with respect to energy) and the coupling describes gain/loss at a given energy level due to inelastic collisions.

- A system of diffusion equations with a strong coupling, from both zeroth order terms and diffusion currents which are now non local: energy exchanges during the collisions induce diffusive effects.

\subsection{Fundamental properties of the diffusivity}

We are naturally led to discuss some properties of the diffusivity $D$. To this end, one introduces the following Hilbert space of vector-valued sequences

$$
\mathbb{H}=\left\{\Phi: \mathbb{N} \longrightarrow \mathbb{R}^{N} ; \sum_{i}\left|\Phi_{i}\right|^{2} \frac{2 \varepsilon i}{m \gamma_{i} B_{i}}<+\infty\right\} .
$$

We shall identify $\ell=\left\{\Phi: \mathbb{N} \rightarrow \mathbb{R}^{N}, \sum_{i}\left|\Phi_{i}\right|^{2} B_{i}^{-1}<\infty\right\}$ with its dual; accordingly, the dual of $\mathbb{H}$ reads

$$
\mathbb{H}^{\prime}=\left\{J: \mathbb{N} \longrightarrow \mathbb{R}^{N} ; \sum_{i}\left|J_{i}\right|^{2} \frac{m \gamma_{i}}{2 \varepsilon i B_{i}}<+\infty\right\},
$$

with the duality relation

$$
\langle J, \Phi\rangle_{\mathbb{H}, \mathbb{H}}=\left(J \sqrt{\frac{m c_{i}}{2 \varepsilon i}}, \Phi \sqrt{\frac{2 \varepsilon i}{m c_{i}}}\right)_{\ell}=\sum_{i} J_{i} \Phi_{i} B_{i}^{-1} .
$$


Lemma 1 Assume (H). Let us set

$$
\mathcal{D}(\Psi, \Phi)=\sum_{i, j} D_{i j} \Psi_{j} \cdot \Phi_{i} B_{i}^{-1} \quad \forall \Phi, \Psi \in \mathbb{H} .
$$

Then,

(i) $\mathcal{D}$ is a bilinear continuous form on $\mathbb{H}$;

(ii) $\mathcal{D}$ is positive-definite (symmetric as soon as $Q_{\theta}$ is a self adjoint operator). Furthermore, there exists a constant $c>0$ such that for all $\Phi \in \mathbb{H}$,

$$
\mathcal{D}(\Phi, \Phi) \geq c\|\Phi\|_{\mathbb{H}}^{2} .
$$

Proof. Assuming (H), one associates to $\Phi$ in the weighted $\ell^{2}$ space $\mathbb{H}$, the function

$$
\begin{aligned}
& \phi: \mathbb{N} \times S^{N-1} \longrightarrow \mathbb{R} \\
& (i, \omega) \quad \longmapsto \phi_{i}(\omega)=v_{i} \cdot \Phi_{i}=\omega \cdot \sqrt{2 \varepsilon i / m} \Phi_{i} .
\end{aligned}
$$

One remarks that $\phi$ lies in $\mathbb{F}_{0}$, and $\|\phi\|_{\mathbb{F}}$ provides a norm equivalent to the natural norm on $\mathbb{H}$ (by using (h2)). Therefore, Proposition 4 allows us to define the mapping

$$
\begin{aligned}
K: \mathbb{H} & \longrightarrow \mathbb{E}_{0} \\
\Phi & \longmapsto K^{\Phi},
\end{aligned}
$$

with $Q^{*}\left(K^{\Phi}\right)_{i}(\omega)=v_{i} \cdot \Phi_{i}=\phi_{i}(\omega)$ i.e. $K^{\Phi}=Q^{*-1}(\phi)$. Clearly, $K$ is a bounded linear operator from $\mathbb{H}$ to $\mathbb{E}$.

With these notations, we have the following relation, which will be proved later on,

$$
B_{j}^{-1} K_{j}^{\Phi}(\omega)=\sum_{i} \chi_{j}^{i}(\omega) \cdot \Phi_{i} B_{i}^{-1} .
$$

From the definition of $D_{i j}$, and (23), we can rewrite $\mathcal{D}$ as follows:

$$
\begin{aligned}
\mathcal{D}(\Psi, \Phi) & =\sum_{i j} D_{i j} \Psi_{j} \cdot \Phi_{i} B_{i}^{-1}=-\sum_{i j} \int_{S^{N-1}} \chi_{j}^{i}(\omega) \cdot \Phi_{i} B_{i}^{-1} v_{j} \cdot \Psi_{j} d \omega \\
& =-\sum_{j} \int_{S^{N-1}}\left(\sum_{i} \chi_{j}^{i}(\omega) \cdot \Phi_{i} B_{i}^{-1}\right) v_{j} \cdot \Psi_{j} d \omega \\
& =-\sum_{j} \int_{S^{N-1}} B_{j}^{-1} K_{j}^{\Phi}(\omega) \psi_{j}(\omega) d \omega .
\end{aligned}
$$

Hence we have

$$
\begin{aligned}
\mathcal{D}(\Psi, \Phi) & =-\sum_{i} \int_{S^{N-1}} \psi_{i}(\omega) Q^{*-1}(\phi)_{i}(\omega) B_{i}^{-1} d \omega \\
& =-\sum_{i}\left(\Psi_{i} \cdot\left(\int_{S^{N-1}} v_{i} K_{i}^{\Phi} d \omega\right) B_{i}^{-1}\right) .
\end{aligned}
$$

INRIA 
The lemma follows, since the first expression recasts as

$$
-\sum_{i} \int_{S^{N-1}} Q^{*}\left(K^{\Psi}\right)_{i}(\omega) K_{i}^{\Phi}(\omega) B_{i}^{-1} d \omega=\mathcal{B}_{\theta}\left(K^{\Phi}, K^{\Psi}\right)
$$

which easily leads to the conclusion by using Corollary 3 .

We are thus left with the task of proving (23). However, this is a simple consequence of the linearity of $Q^{*}$, which implies that summation over $i$ commutes with the action of this operator. We are thus able to show that $Q^{*}$ acts on the right hand side of (23) as follows

$$
\begin{aligned}
Q^{*}\left(\sum_{i} B \cdot \chi^{i} \cdot \Phi_{i} B_{i}^{-1}\right)_{j} & =\sum_{i} Q^{*}\left(B \cdot \chi^{i}\right)_{j} \cdot \Phi_{i} B_{i}^{-1} \\
& =\sum_{i}^{i} v_{i} B_{i} \delta_{i j} \cdot \Phi_{i} B_{i}^{-1}=v_{j} \cdot \Phi_{j}=\phi_{j}=Q^{*}\left(K^{\Phi}\right)_{j} .
\end{aligned}
$$

From Lemma 1, we can deduce the following claim which will give a precise meaning to the current relation (19). It will play a key role in our rigorous analysis.

Corollary 4 We can define a linear, continuous and invertible mapping $\mathcal{J}: \mathbb{H} \longrightarrow \mathbb{H}^{\prime}$ such that, for any $\Phi$ and $\Psi$ in $\mathbb{H}$ one has

$$
\mathcal{D}(\Phi, \Psi)=-(\mathcal{J}(\Phi), \Psi)_{\ell}=-\sum_{i} \mathcal{J}(\Phi)_{i} \Psi_{i} B_{i}^{-1} .
$$

Proof. For any $\Phi \in \mathbb{H}$, the Riesz theorem defines a unique $\mathcal{J}(\Phi) \in \mathbb{H}^{\prime}$, such that $\mathcal{D}(\Phi, \Psi)=$ $-(\mathcal{J}(\Phi), \Psi)_{\boldsymbol{\ell}}$ for any $\Psi \in \mathbb{H}$. The corresponding mapping $\mathcal{J}: \mathbb{H} \longrightarrow \mathbb{H}^{\prime}$ is of course linear and continuous. Conversely, let $J \in \mathbb{H}^{\prime}$. The Lax-Milgram theorem applies to solve the variational problem

find $\Phi \in \mathbb{H}$ such that, for all $\Psi \in \mathbb{H}$ one has $\mathcal{D}(\Phi, \Psi)=-(J, \Psi)_{\ell}$.

By Proposition 4, this problem admits a unique solution $\Phi \in \mathbb{H}$ which satisfies, by its definition, $\mathcal{J}(\Phi)=J$.

This statement will allow us to interpret the current equation (19) in a duality sense by $J=\mathcal{J}\left(\nabla_{x} \rho\right)$. Accordingly, one expects that $J$ lies in $L^{2}\left(\mathbb{R}_{t}^{+} \times \mathbb{R}_{x}^{N} ; \mathbb{H}^{\prime}\right)$ and $\nabla_{x} \rho \in$ $L^{2}\left(\mathbb{R}_{t}^{+} \times \mathbb{R}_{x}^{N} ; \mathbb{H}\right)$. It is worth remarking this gain in "regularity", both in the space and energy variables on the density, since this property is not guaranteed in general for $\rho^{\eta}$, for $\eta>0$. This is an usual fact when dealing with diffusion approximation, see for instance [29], [20]; the noticeable point here is that this effect also applies to the energy variable.

\section{Rigorous derivation}

This section is devoted to the rigorous study of the asymptotic behaviour as $\eta$ goes to 0 of the solution $f^{\eta}$ of the kinetic equation

$$
\left\{\begin{array}{l}
\partial_{t} f_{i}^{\eta}+\frac{1}{\eta} v_{i} \cdot \nabla_{x} f_{i}^{\eta}=\frac{1}{\eta^{2}}\left(Q_{\theta}\left(f^{\eta}\right)\right)_{i} \quad \text { in } \mathbb{R}_{t}^{+} \times \mathbb{R}_{x}^{N} \times \mathbb{N}_{i} \times S_{\omega}^{N-1} \\
f^{\eta}(t=0)=F_{I}^{\eta}
\end{array}\right.
$$

$\mathrm{RR} \mathrm{n}^{\circ} 4302$ 
In order to take into account time and space variables, one needs to define again some functional spaces, based on the previous ones. We set

$$
\mathcal{L}^{2}=\left\{f: \mathbb{R}_{x}^{N} \times \mathbb{N}_{i} \times S_{\omega}^{N-1} \longrightarrow \mathbb{R}, \sum_{i} \int_{\mathbb{R}^{N}} \int_{S^{N-1}} f_{i}^{2} B_{i}^{-1} d \omega d x<\infty\right\}
$$

It is nothing but the $L^{2}$ space on $\mathbb{R}^{N} \times \mathbb{N} \times S^{N-1}$ endowed with the measure $d x \otimes\left(B_{i}^{-1} d i\right) \otimes d \omega$, where $d i$ stands for the counting measure on $\mathbb{N}$. Accordingly, we also set

$$
\mathcal{E}=\left\{f: \mathbb{R}_{x}^{N} \times \mathbb{N}_{i} \times S_{\omega}^{N-1} \longrightarrow \mathbb{R}, \sum_{i} \int_{\mathbb{R}^{N}} \int_{S^{N-1}} f_{i}^{2} \frac{\gamma_{i}}{B_{i}} d \omega d x<\infty\right\}=L^{2}\left(\mathbb{R}^{N} ; \mathbb{E}\right)
$$

and

$$
\mathcal{F}=\left\{f: \mathbb{R}_{x}^{N} \times \mathbb{N}_{i} \times S_{\omega}^{N-1} \longrightarrow \mathbb{R}, \sum_{i} \int_{\mathbb{R}^{N}} \int_{S^{N-1}} f_{i}^{2} \frac{1}{\gamma_{i} B_{i}} d \omega d x<\infty\right\}=L^{2}\left(\mathbb{R}^{N} ; \mathbb{F}\right) .
$$

Similarly, for vector-valued macroscopic quantities, independent on the angular variable, we set

$$
\mathcal{H}=\left\{\Phi: \mathbb{R}_{x}^{N} \times \mathbb{N}_{i} \longrightarrow \mathbb{R}^{N}, \sum_{i} \int_{\mathbb{R}^{N}}\left|\Phi_{i}\right|^{2} \frac{2 \varepsilon i}{m \gamma_{i} B_{i}} d x<\infty\right\}=L^{2}\left(\mathbb{R}^{N} ; \mathbb{H}\right)
$$

and

$$
\mathcal{H}^{\prime}=\left\{J: \mathbb{R}_{x}^{N} \times \mathbb{N}_{i} \longrightarrow \mathbb{R}, \sum_{i} \int_{\mathbb{R}^{N}}\left|J_{i}\right|^{2} \frac{m \gamma_{i}}{2 \varepsilon i B_{i}} d x<\infty\right\}=L^{2}\left(\mathbb{R}^{N} ; \mathbb{H}^{\prime}\right)
$$

which is the dual of $\mathcal{H}$ when one identifies $L^{2}\left(\mathbb{R}^{N}, \mathfrak{l}\right)=L^{2}\left(\mathbb{R}^{N} \times \mathbb{N}, d x \otimes B_{i}^{-1} d i\right)$ with its dual, namely

$$
\langle J, \Phi\rangle_{\mathcal{H}^{\prime}, \mathcal{H}}=\sum_{i} \int_{\mathbb{R}^{N}} \Phi_{i} \cdot J_{i} B_{i}^{-1} d x .
$$

We shall not detail the existence theory for the transport equation (25). With some maybe stronger assumptions on the cross-sections, this can be done by means of semi-groups theory. We can also use some monotonicity argument, following R. Petterson [36]. Instead, we shall assume from now on that there exists a function $f^{\eta} \in C^{0}\left(\mathbb{R}^{+} ; \mathcal{L}^{2}\right) \cap L^{\infty}\left(\mathbb{R}^{+} ; \mathcal{E}\right)$ satisfying the following weak formulation of (25)

$$
\begin{aligned}
& -\int f^{\eta} \partial_{t} \varphi B^{-1} d \mu-\frac{1}{\eta} \int f^{\eta} v \cdot \nabla_{x} \varphi B^{-1} d \mu+\left[\int f^{\eta} \varphi B^{-1} d \nu\right]_{0}^{T} \\
& =\frac{1}{\eta^{2}} \int Q_{\theta}\left(f^{\eta}\right) \varphi B^{-1} d \mu .
\end{aligned}
$$

Here, $d \mu$ indicates integration over $(0, T) \times \mathbb{R}^{N} \times \mathbb{N} \times S^{N-1}$ with the measure $d t \otimes d x \otimes d i \otimes d \omega$ while $d \nu$ has the same meaning on $\mathbb{R}^{N} \times \mathbb{N} \times S^{N-1}$ with the measure $d x \otimes d i \otimes d \omega$. Relation (26) holds for any $\varphi$ in an appropriate space of admissible test functions $\mathfrak{D}$. In particular, it makes sense for $\varphi \in L_{l o c}^{1}\left(\mathbb{R}^{+} ; \mathcal{E}\right)$ such that $\partial_{t} \varphi$ and $\nabla_{x} \cdot(v \varphi)$ belong to $L_{l o c}^{1}\left(\mathbb{R}^{+} ; \mathcal{L}^{2}\right)$. It is worth remarking that a sequence defined by a finite number of non-zero functions $\varphi_{i}(t, x, \omega)$ lying in $C_{0}^{1}\left([0, T] \times \mathbb{R}^{N} ; L^{\infty}\left(S^{N-1}\right)\right)$ is admissible. 
REMARK 8 If one assumes that $0<\gamma_{i} \leq C<+\infty$, we obtain the inclusions $\mathbb{F} \subset \mathbb{L} \subset \mathbb{E}$, and therefore $Q_{\theta}$ can be viewed as a bounded operator on $\mathcal{L}^{2}$, with values in $\mathcal{L}^{2}$, and existenceuniqueness is easy to be established. Of course, the problem for $\eta>0$ fixed and most of our convergence analysis become easier if one deals with a finite number of energy groups.

Then, we can now state our main result.

Theorem 1 Suppose $(H)$ and let $F_{I}^{\eta}$ be bounded in $\mathcal{L}^{2}$. Then, $\rho^{\eta}=\int_{S^{N-1}} f^{\eta} d \omega$ converges weakly-* in $L^{\infty}\left(0, T ; L^{2}\left(\mathbb{R}^{N} \times \mathbb{N} ; B^{-1} d i \otimes d x\right)\right)$ (and in $C^{0}\left([0, T], L^{2}\left(\mathbb{R}^{N} \times \mathbb{N} ; B^{-1} d i \otimes d x\right)-\right.$ weak)) to $\rho$ and $J^{\eta}=\int_{S^{N-1}} \sqrt{2 \varepsilon i / m} \omega f^{\eta} d \omega$ converges weakly-* in $L^{2}\left(0, T ; \mathcal{H}^{\prime}\right)$ to $J$ (see (27) below). These limits satisfy

$$
\left\{\begin{array}{l}
\partial_{t} \rho+\operatorname{div}_{x} J=0, \\
\nabla_{x} \rho \in L^{2}(0, T ; \mathcal{H}), \quad \text { and } \quad \mathcal{J}\left(\nabla_{x} \rho\right)=J .
\end{array}\right.
$$

The proof naturally falls into four steps.

Step 1: A priori estimates and weak convergences

The main estimates on $f^{\eta}$ are obtained by formally multiplying equation (25) by $f^{\eta} / B$ and integrating. It yields

$$
\left\|f^{\eta}(t)\right\|_{\mathcal{L}^{2}}^{2}+\frac{2}{\eta^{2}} \int_{0}^{t} \int_{\mathbb{R}^{N}} \mathcal{B}_{\theta}\left(f^{\eta}, f^{\eta}\right) d x d s=\left\|F_{I}^{\eta}\right\|_{\mathcal{L}^{2}}^{2} .
$$

Therefore, the coercivity of $\mathcal{B}_{\theta}$ allows us to estimate $\left\|f^{\eta}\right\|_{\mathcal{L}^{2}}$ and $\frac{1}{\eta} N\left(f^{\eta}-\rho^{\eta}\right)=N\left(g^{\eta}\right)$ and thus leads to the following statement.

Lemma 2 Suppose that $(H)$ holds and let $F_{I}^{\eta}$ be bounded in $\mathcal{L}^{2}$. Then,

i) $f^{\eta}$ is bounded in $L^{\infty}\left(\mathbb{R}_{t}^{+} ; \mathcal{L}^{2}\right)$, with $\left\|f^{\eta}(t)\right\|_{\mathcal{L}^{2}}^{2} \leq\left\|F_{I}^{\eta}\right\|_{\mathcal{L}^{2}}^{2} \leq C$.

ii) $g^{\eta}=\left(f^{\eta}-\rho^{\eta}\right) / \eta$ is bounded in $L^{2}\left(\mathbb{R}_{t}^{+} ; \mathcal{E}\right)$,

iii) $\rho_{i}^{\eta}(t, x)=\int_{S^{N-1}} f_{i}^{\eta}(t, x, \omega) d \omega$ is bounded in $L^{\infty}\left(\mathbb{R}_{t}^{+} ; L^{2}\left(\mathbb{R}^{N} \times \mathbb{N}, d x \otimes B_{i}^{-1} d i\right)\right)$,

iv) $J_{i}^{\eta}(t, x)=1 / \eta \int_{S^{N-1}} v_{i} f^{\eta}(t, x, \omega) d \omega=\int_{S^{N-1}}(2 \varepsilon i) / m \omega g^{\eta}(t, x, \omega) d \omega$ is bounded in $L^{\infty}\left(\mathbb{R}_{t}^{+} ; \mathcal{H}^{\prime}\right)$.

Proof. It remains to establish the bounds on the macroscopic quantities. Clearly, one has

$$
\sum_{i} \int_{\mathbb{R}^{N}}\left|\rho_{i}^{\eta}\right|^{2} B_{i}^{-1} d x=\sum_{i} \int_{\mathbb{R}^{N}}\left|\int_{S^{N-1}} f_{i}^{\eta} d \omega\right|^{2} B_{i}^{-1} d x \leq\left\|f^{\eta}\right\|_{\mathcal{L}^{2}}
$$

Next, the current satisfies

$$
\begin{aligned}
\sum_{i} \int_{\mathbb{R}^{+}} \int_{\mathbb{R}^{N}}\left|J_{i}^{\eta}\right|^{2} \frac{m \gamma_{i}}{2 \varepsilon i B_{i}} d x d t & =\sum_{i} \int_{\mathbb{R}^{+}} \int_{\mathbb{R}^{N}}\left|\int_{S^{N-1}} \sqrt{\frac{2 \varepsilon i}{m}} \omega g_{i}^{\eta} d \omega\right|^{2} \frac{m \gamma_{i}}{2 \varepsilon i B_{i}} d x d t \\
& \leq \sum_{i} \int_{\mathbb{R}^{+}} \int_{\mathbb{R}^{N}} \int_{S^{N-1}}\left|g_{i}^{\eta}\right|^{2} \frac{\gamma_{i}}{B_{i}} d \omega d x d t \\
& \leq \int_{\mathbb{R}^{+}} \int_{\mathbb{R}^{N}} N\left(g^{\eta}\right)^{2} d x d t
\end{aligned}
$$

RR $n^{\circ} 4302$ 
and the boundedness of $J^{\eta}$ is a consequence of ii).

Possibly at the cost of extracting subsequences, we can assume that

$$
\begin{cases}f^{\eta} \rightarrow_{*} f & \text { weakly } * \text { in } L^{\infty}\left(\mathbb{R}_{t}^{+} ; \mathcal{L}^{2}\right), \\ g^{\eta} \rightarrow_{*} g & \text { weakly } * \text { in } L^{2}\left(\mathbb{R}_{t}^{+} ; \mathcal{E}\right), \\ \rho^{\eta} \rightarrow_{*} \rho & \text { weakly } * \text { in } L^{\infty}\left(\mathbb{R}_{t}^{+} ; L^{2}\left(\mathbb{R}^{N} \times \mathbb{N}, d x \otimes B_{i}^{-1} d i\right)\right), \\ J^{\eta} \rightarrow_{*} J & \text { weakly } * \text { in } L^{2}\left(\mathbb{R}_{t}^{+} ; \mathcal{H}^{\prime}\right) .\end{cases}
$$

This means, respectively

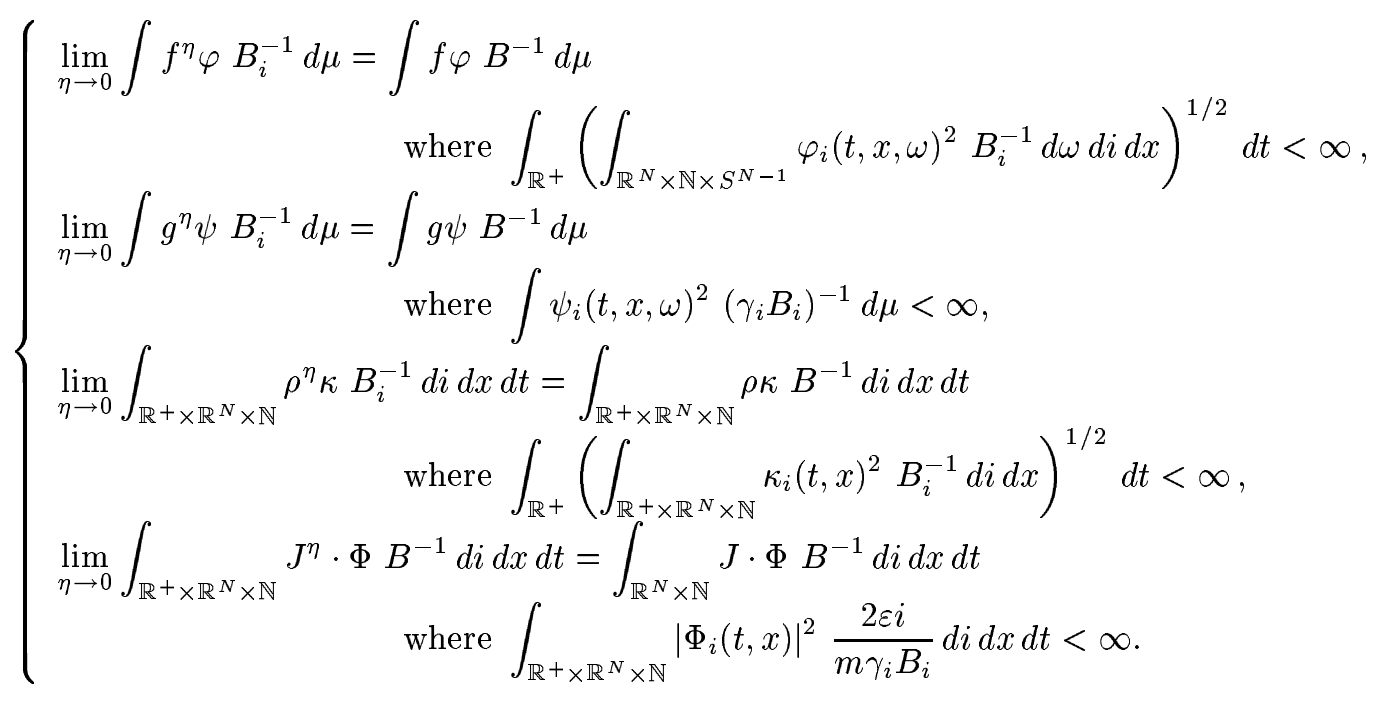

Choosing, with the above properties, $\varphi_{i}(t, x, \omega)=\kappa_{i}(t, x)$ and $\psi_{i}(t, x, \omega)=(2 \varepsilon i / m)^{1 / 2} \omega$. $\Phi_{i}(t, x)$, we realize that

$$
\rho=\int_{S^{N-1}} f d \omega, \quad J=\int_{S^{N-1}} \sqrt{\frac{2 \varepsilon i}{m}} \omega g d \omega
$$

holds. In fact, we obviously have $f=\rho$ by taking the limit in the distributional sense in $f^{\eta}=\rho^{\eta}+\eta g^{\eta}$.

Step 2: Continuity equation

We wish to establish the following claim.

Lemma 3 For any $i$ the continuity relation

$$
\partial_{t} \rho_{i}+\operatorname{div}_{x} J_{i}=0
$$

holds in $\mathcal{D}^{\prime}\left((0, T) \times \mathbb{R}^{N}\right)$.

Proof. We use in (26) the test function $\kappa_{i} B_{i} \zeta(t, x)$ with $\kappa_{i} \in \ell^{\infty}$ (precisely $\kappa_{i}=\delta_{i j}$ ), and $\zeta \in C_{0}^{\infty}\left((0, T) \times \mathbb{R}^{N}\right)$; then we take the limit $\eta \rightarrow 0$. 
REMARK 9 Under suitable hypotheses, one could also rigorously derive the inelastic term (22). Actually, if $\sigma_{i j}\left(\omega, \omega^{\prime}\right)$ is such that $Q_{\theta}^{\text {inel }}$ is bounded in $\mathbb{L}$, a-priori estimate can still be derived by means of an application of Gronwall's lemma when $Q_{\theta}=Q_{\theta}^{e l}+\eta^{2} Q_{\theta}^{\text {inel }}$.

Step 3: Current equation

According to our discussion in the previous section, the expected current equation $J=$ $-\sum_{j} D_{i j} \nabla_{x} \rho_{j}$ has to be understood in the dual sense $\mathcal{J}\left(\nabla_{x} \rho\right)=J$ which means that

$$
\mathcal{D}\left(\nabla_{x} \rho, \Phi\right)=-\langle J, \Phi\rangle
$$

holds for any $\Phi \in L^{2}\left(\mathbb{R}^{+}, \mathcal{H}\right)$. Of course, the notation now takes into account the time and space variables. Precisely, the right hand side reads

$$
\int J \cdot \Phi B^{-1} d i d x d t=\int g v \cdot \Phi B^{-1} d \mu .
$$

Since we can associate to $\Phi \in L^{2}\left(\mathbb{R}^{+}, \mathcal{H}\right)$ a unique $K^{\Phi} \in L^{2}\left(\mathbb{R}^{+}, \mathcal{E}\right)$ (with null average) such that $Q^{*}\left(K^{\Phi}\right)=v \cdot \Phi$, we get

$$
(J, \Phi)=\int g Q^{*}\left(K^{\Phi}\right) B^{-1} d \mu .
$$

On the other hand, the left hand side in (28) is

$$
-\int \nabla_{x} \rho \cdot v K^{\Phi} B^{-1} d \mu=-\int_{\mathbb{R} \times \mathbb{N} \times \mathbb{R}^{N}} \nabla_{x} \rho\left(\int_{S^{N-1}} v K^{\Phi} d \omega\right) B_{i}^{-1} d i d x d t
$$

by using (23). Then, (28) reduces to

$$
\int g Q^{*}\left(K^{\Phi}\right) B^{-1} d \mu=\int_{\mathbb{R} \times \mathbb{N} \times \mathbb{R}^{N}} \nabla_{x} \rho\left(\int_{S^{N-1}} v K^{\Phi} d \omega\right) B^{-1} d i d x d t
$$

In order to justify equality (28), it thus would be tempting to use $\varphi=K^{\Phi}$ as test function in (26). However, difficulties arise when we ask for such a function to belong to the admissible set $\mathfrak{D}$. We note that, for $\Phi \in L^{2}(0, T ; \mathcal{H})$, we naturally have $K^{\Phi} \in L^{2}(0, T ; \mathcal{E})$ and $\int_{S^{N-1}} v K^{\Phi} d \omega \in L^{2}\left(0, T ; \mathcal{H}^{\prime}\right)$. However we recall that it is required that $\nabla_{x} \cdot(v \varphi) \in$ $L^{2}\left(0, T ; \mathcal{L}^{2}\right)$ for $\varphi$ to be an admissible test function. Space regularity is not a real difficulty, but it is not clear how it can be guaranteed that $v K^{\Phi}$ belongs to $L^{2}\left(0, T ; \mathcal{L}^{2}\right)$. Except in some very particular cases (for instance if $2 \varepsilon i \leq C \gamma_{i}$ which implies that $\mathcal{H}^{\prime}$ embeds to $\ell$ or in some isotropic case as in [19]), since $Q_{\theta}^{-1}$ mixes all the energy levels, it seems that a truncation of $\Phi$ on the high levels does not give such a control and it is not clear at all that the set $\left\{\Phi \in L^{2}(0, T ; \mathcal{H}), \nabla_{x} \cdot v K^{\Phi} \in L^{2}\left(0, T ; \mathcal{L}^{2}\right)\right\}$ is not empty in the general case. This difficulty leads to some technical restrictions on the cross-sections in [19].

On the other hand, if we are able to prove that $\nabla_{x} \rho \in L^{2}(0, T ; \mathcal{H})$, then, the relation (29) makes sense provided $\Phi \in L^{2}(0, T ; \mathcal{H})$ and does not require further property on $\Phi$. This motivates our strategy of proof: First, we establish an approximate current equation, from which we will be able to deduce that $\nabla_{x} \rho \in L^{2}(0, T ; \mathcal{H})$; and then we derive the current equation (29). We are thus led to the main statement of the step. 
Proposition 5 We have $\nabla_{x} \rho \in L^{2}\left(0, T ; \mathcal{H}\right.$ ), and the limit $J$ of $J^{\eta}$ satisfies (29).

Proof. Let $\Phi$ be a test function in $L^{2}(0, T ; \mathcal{H})$ compactly supported with respect to time in $(0, T)$. Moreover, one assumes that $\partial_{t} \Phi \in L^{2}(0, T ; \boldsymbol{l})$ and $\nabla_{x} \Phi \in L^{2}(0, T ; \mathcal{H})$. This can be obtained by regularizing in time and space and eventually truncating in energy a function in $L^{2}(0, T ; \mathcal{H})$. Then, $Q_{\theta}^{*-1}(v \cdot \Phi)=K^{\Phi} \in L^{2}(0, T ; \mathcal{E})$ (recall that $K^{\Phi}$ depends on $\omega$, though $\Phi$ does not). We introduce the function $\mu^{(n)}$ defined on $\mathbb{N}$ by

$$
\mu_{i}^{(n)}= \begin{cases}1 & \text { if } i \leq n, \\ 0 & \text { if } i>n .\end{cases}
$$

The parameter $n$ will help us in approximating (29). Then, we consider the admissible test function $\varphi_{i}(t, x, \omega)=\mu_{i}^{(n)} K_{i}^{\Phi}(t, x, \omega) \in L^{2}(0, T ; \mathcal{E})$. We obtain from (26)

$-\int f^{\eta} v \cdot \nabla_{x}\left(\mu^{(n)} K^{\Phi}\right) B^{-1} d \mu-\eta \int f^{\eta} \partial_{t}\left(\mu^{(n)} K^{\Phi}\right) B^{-1} d \mu=\frac{1}{\eta} \int Q_{\theta}\left(f^{\eta}\right) \mu^{(n)} K^{\Phi} B^{-1} d \mu$.

The right hand side gives

$$
\int Q_{\theta}\left(g^{\eta}\right) \mu^{(n)} K^{\Phi} B^{-1} d \mu=\int g^{\eta} Q_{\theta}^{*}\left(\mu^{(n)} K^{\Phi}\right) B^{-1} d \mu,
$$

while the left hand side is rewritten

$$
\begin{aligned}
& -\int \rho^{\eta} \mu^{(n)} v \cdot \nabla_{x} K^{\Phi} B^{-1} d \mu \\
& -\eta\left(\int f^{\eta} \partial_{t}\left(\mu^{(n)} K^{\Phi}\right) B^{-1} d \mu+\int g^{\eta} \mu^{(n)} v \cdot \nabla_{x} K^{\Phi} B^{-1} d \mu\right) .
\end{aligned}
$$

Since all the sums are actually finite, there is no trouble in applying convergences (27), $n$ being fixed. The left hand side in (30) tends, as $\eta$ goes to 0 , towards

$$
-\int \rho \nabla_{x} \cdot\left(v_{j} \mu^{(n)} v K^{\Phi}\right) B^{-1} d \mu=-\int_{\mathbb{R}^{+} \times \mathbb{R}^{N} \times \mathbb{N}}\left(\mu^{(n)} \rho\right) \nabla_{x} \cdot\left(\int_{S^{N-1}} v K^{\Phi} d \omega\right) B^{-1} d i d x d t .
$$

Moreover, the right hand side in (30) becomes

$$
\int g^{\eta} Q_{\theta}^{*}\left(\mu^{(n)} K^{\Phi}\right) B^{-1} d \mu \longrightarrow \int g Q_{\theta}^{*}\left(\mu^{(n)} K^{\Phi}\right) B^{-1} d \mu .
$$

Thus, we are led to the equality

$$
-\int_{\mathbb{R}^{+} \times \mathbb{R}^{N} \times \mathbb{N}}\left(\mu^{(n)} \rho\right) \nabla_{x} \cdot\left(\int_{S^{N-1}} v K^{\Phi} d \omega\right) B^{-1} d i d x d t=\int g Q_{\theta}^{*}\left(\mu^{(n)} K^{\Phi}\right) B^{-1} d \mu,
$$

which appears as an approximate form of (28). We wish to conclude by letting $n$ go to $\infty$. Let us introduce the linear mapping $J^{(n)}$ defined on the set

$$
\left\{\Phi \in L^{2}(0, T ; \mathcal{H}), \nabla_{x} \cdot\left(\int_{S^{N-1}} v K^{\Phi} d \omega\right) \in L^{2}\left(0, T ; \mathcal{H}^{\prime}\right)\right\}
$$

INRIA 
by

$$
J^{(n)}(\Phi)=-\int_{\mathbb{R}^{+} \times \mathbb{R}^{N} \times \mathbb{N}}\left(\mu^{(n)} \rho\right) \nabla_{x} \cdot\left(\int_{S^{N-1}} v K^{\Phi} d \omega\right) B^{-1} d i d x d t,
$$

which corresponds to a weak definition of $-\mathcal{D}\left(\mu^{(n)} \nabla_{x} \rho, \Phi\right)$. Note that this set is obviously dense in $L^{2}(0, T ; \mathcal{H})$ (it is only concerned with space regularization since $\int_{S^{N-1}} v K^{\Phi} d \omega$ naturally lies in $\left.L^{2}\left(0, T ; \mathcal{H}^{\prime}\right)\right)$. Furthermore, $(31)$ says that one actually has

$$
J^{(n)}(\Phi)=\int g Q_{\theta}^{*}\left(\mu^{(n)} K^{\Phi}\right) B^{-1} d \mu .
$$

One deduces that

$$
\left|J^{(n)}(\Phi)\right| \leq C\|g\|_{L^{2}(0, T ; \mathcal{E})}\|\Phi\|_{L^{2}(0, T ; \mathcal{H})}
$$

and $J^{(n)}$ is a bounded sequence of continuous linear forms defined on the whole space $L^{2}(0, T ; \mathcal{H})$. According to Corollary 4 , we can associate a sequence $\Theta^{(n)}$ satisfying

$$
\left\|\Theta^{(n)}\right\|_{L^{2}(0, T ; \mathcal{H})} \leq C\|g\|_{L^{2}(0, T ; \mathcal{E})},
$$

and

$$
J^{(n)}(\Phi)=-\mathcal{D}\left(\Theta^{(n)}, \Phi\right)=\int_{\mathbb{R}^{+} \times \mathbb{R}^{N} \times \mathbb{N}} \Theta_{i}^{(n)}(t, x)\left(\int_{S^{N-1}} v_{i} K_{i}^{\Phi}(t, x, \omega) d \omega\right) B_{i}^{-1} d i d x d t,
$$

for any $\Phi \in L^{2}\left(0, T ; \mathcal{H}\right.$ ) (where we used $(24)$ ). Then, coming back to $(31), \Theta^{(n)}$ is a bounded sequence in $L^{2}(0, T ; \mathcal{H})$ which coincides with $\mu^{(n)} \nabla_{x} \rho$ as a linear form on the subset $\left\{\int_{S^{N-1}} v K^{\Phi} d \omega, \Phi \in L^{2}(0, T ; \mathcal{H})\right\} \subset L^{2}\left(0, T ; \mathcal{H}^{\prime}\right)$. Hence, we will conclude that the limit of $\Theta^{(n)}$ defines $\nabla_{x} \rho$ as element of $L^{2}(0, T ; \mathcal{H})$ if we are able to prove the following claim.

Lemma 4 The set $\mathbb{K}=\left\{\int_{S^{N-1}} v K^{\Phi} d \omega, \Phi \in \mathbb{H}\right\}$ is dense in $\mathbb{H}^{\prime}$.

Lemma 4 yields $\mu^{(n)} \nabla_{x} \rho=\Theta^{(n)}$ in $L^{2}(0, T ; \mathcal{H})$, and therefore $\mu^{(n)} \nabla_{x} \rho$ is bounded in this space, uniformly with respect to $n$. Since the sequence converges towards $\nabla_{x} \rho$ as $n \rightarrow \infty$ in a distributional sense, we deduce that $\mu^{(n)} \nabla_{x} \rho$ converges weakly towards $\nabla_{x} \rho$ in $L^{2}(0, T, \mathcal{H})$.

We end the proof by taking the limit $n \rightarrow \infty$ in the equality (31); we finally get

$$
-\mathcal{D}\left(\nabla_{x} \rho, \Phi\right)=\int g v \cdot \Phi B^{-1} d \mu=\int_{\mathbb{R}^{+} \times \mathbb{R}^{N} \times \mathbb{N}} J \cdot \Phi B^{-1} d i d x d t .
$$

Now, it remains to justify Lemma 4 .

Proof of Lemma 4. It is equivalent to prove that the orthogonal set of $\mathbb{K}$ is reduced to 0 in $\mathbb{H}$. Let $\Theta \in \mathbb{H}$ satisfy

$$
\left(\Theta, \int_{S^{N-1}} v K^{\Phi} d \omega\right)_{\mathbb{L}}=0, \quad \forall \Phi \in \mathbb{H} .
$$


Then, by (24), this reads $\mathcal{D}(\Theta, \Phi)=0$ for all $\Phi \in \mathbb{H}$, which yields $\Theta=0$ thanks to the coercivity of the bilinear form $\mathcal{D}$.

Step 4: Compactness in time.

We would like to recover as initial data $\rho_{I}$ for the limit problem a function depending on the behaviour of the sequence of data $F_{I}^{\eta}$ for the kinetic equation. Of course this relies on a compactness in time property, at least for some weak topology. On the other hand, it can be shown a uniqueness result for the limit equation in the class of continuous functions with value in $L^{2}\left(\mathbb{R}^{N} \times \mathbb{N} ; B^{-1} d i \otimes d x\right)$ and having the gradient in $L^{2}(0, T ; \mathcal{H})$. In turn, of course, the whole sequence $\rho^{\eta}$ will converge to this $\rho$.

Lemma 5 The sequence $\left(\rho^{\eta}\right)_{\eta>0}$ is sequentially compact in $C^{0}\left([0, T] ; L^{2}\left(\mathbb{R}^{N} \times \mathbb{N}, B_{i}^{-1} d i \otimes\right.\right.$ $d x)$-weak). In particular, there exists a sequence $\left(\rho^{\eta_{n}}\right)_{n \in \mathbb{N}}$ such that for any $\phi \in L^{2}\left(\mathbb{R}^{N} \times\right.$ $\left.\mathbb{N}, B_{i}^{-1} d i \otimes d x\right)$

$$
\int_{\mathbb{R}^{N}} \rho^{\eta_{n}} \phi B^{-1} d i d x \longrightarrow \int_{\mathbb{R}^{N}} \rho \phi B^{-1} d i d x
$$

as $n \rightarrow \infty$ in $C^{0}([0, T])$.

Proof. First, let $\psi \in L^{2}\left(\mathbb{R}^{N} \times \mathbb{N},\left(1+i / \gamma_{i}\right) B_{i}^{-1} d i \otimes d x\right)$ with $\nabla_{x} \psi \in \mathcal{H}$. Looking at the continuity equation, one gets

$$
\begin{aligned}
\int_{\mathbb{R}^{N}} \rho^{\eta}(t) \psi B^{-1} d i d x-\int_{\mathbb{R}^{N}} \rho^{\eta}(s) \psi B^{-1} d i d x & =\int_{0}^{t}\left(J^{\eta}, \nabla_{x} \psi\right)_{\mathcal{H}^{\prime}, \mathcal{H}} d \sigma \\
& \leq C\left\|J^{\eta}\right\|_{L^{2}\left(0, T ; \mathcal{H}^{\prime}\right)} \sqrt{|t-s|}\left\|\nabla_{x} \psi\right\|_{\mathcal{H}} .
\end{aligned}
$$

The bound iv) in Proposition 2 combined to the Arzela-Ascoli theorem allows us to deduce that $\left(\int \rho^{\eta}(t) \psi B^{-1} d i d x\right)_{\eta>0}$ lies in a compact set in $C^{0}([0, T])$. By considering finite series and space regularization, one sees that the set of the $\psi$ 's used above is dense into $L^{2}\left(\mathbb{R}^{N} \times \mathbb{N}, B_{i}^{-1} d i \otimes d x\right)$. Then, let $\phi \in L^{2}\left(\mathbb{R}^{N} \times \mathbb{N}, B_{i}^{-1} d i \otimes d x\right)$. We deduce that, for any $\varepsilon>0$,

$$
\left(\int_{\mathbb{R}^{N}} \rho^{\eta}(t) \phi B^{-1} d i d x\right)_{\eta>0} \subset B(0, \varepsilon)+\text { Compact Set } \quad \text { in } C^{0}([0, T]) .
$$

Hence, $\left(\int \rho^{\eta}(t) \phi B^{-1} d i d x\right)_{\eta>0}$ also lies in a compact set in $C^{0}([0, T])$. We conclude by using the separability of $L^{2}\left(\mathbb{R}^{N} \times \mathbb{N}, B_{i}^{-1} d i \otimes d x\right)$ and a Cantor argument.

Lemma 5 says that we recover as initial data for the limit equation the limit, in the weak $L^{2}\left(\mathbb{R}^{N} \times \mathbb{N}, B_{i}^{-1} d i \otimes d x\right)$ sense, of $\rho_{I}^{\eta}=\int_{S^{N-1}} F_{I}^{\eta} d \omega$. Moreover, classical reasoning gives the continuity in time for the limit problem, see for instance [16].

Lemma 6 The limit $\rho$ is a continuous function on $[0, T]$ with values in $L^{2}\left(\mathbb{R}^{N} \times \mathbb{N}, B_{i}^{-1}\right.$ di $\otimes$ $d x)$; and it satisfies

$$
\frac{d}{d t}\|\rho\|_{L^{2}}^{2}=-2 \int_{\mathbb{R}^{N}} \mathcal{D}\left(\nabla_{x} \rho, \nabla_{x} \rho\right) d x
$$

in $\mathcal{D}^{\prime}(] 0, T[)$. 
Proof. The point relies on the following facts:

$$
\left\{\begin{array}{l}
\rho \in L^{\infty}\left(\mathbb{R}^{+} ; L^{2}\left(\mathbb{R}^{N} \times \mathbb{N}, B_{i}^{-1} d i \otimes d x\right)\right), \\
\rho \in C^{0}\left([0, T] ; L^{2}\left(\mathbb{R}^{N} \times \mathbb{N}, B_{i}^{-1} d i \otimes d x\right)-\text { weak }\right), \\
\nabla_{x} \rho \in L^{2}\left(\mathbb{R}^{+} ; \mathcal{H}\right) .
\end{array}\right.
$$

In turn, the equation (21) implies that $\partial_{t} \rho$ reads as the space derivative of an element of $L^{2}\left(\mathbb{R}^{+} ; \mathcal{H}^{\prime}\right)$. This legitimates the product with $\rho$. Indeed, by regularization (in time) and truncation (in energy), we can construct a sequence $\rho^{(n)}$ which lies in $C^{\infty}\left([0, T] ; \mathcal{H} \cap L^{2}\left(\mathbb{R}^{N} \times\right.\right.$ $\left.\left.\mathbb{N}, B_{i}^{-1} d i \otimes d x\right)\right)$, with $\partial_{t} \rho^{(n)} \in C^{\infty}\left([0, T] ; \mathcal{H}^{\prime} \cap L^{2}\left(\mathbb{R}^{N} \times \mathbb{N}, B_{i}^{-1} d i \otimes d x\right)\right)$ and satisfying

$$
\left\{\begin{array}{l}
\rho^{(n)} \rightarrow \rho \quad \text { in } L^{2}\left(0, T ; L^{2}\left(\mathbb{R}^{N} \times \mathbb{N}, B_{i}^{-1} d i \otimes d x\right)\right) \\
\partial_{t} \rho^{(n)} \rightarrow \partial_{t} \rho \quad \text { in } L^{2}\left(0, T ; \nabla_{x} \cdot\left(\mathcal{H}^{\prime}\right)\right)
\end{array}\right.
$$

as $n$ goes to $\infty$. When dealing with regular functions, one has clearly

$$
\frac{d}{d t}\left\|\rho^{(n)}\right\|_{L^{2}}^{2}=2\left(\partial_{t} \rho^{(n)}, \rho^{(n)}\right)_{L^{2}}=2\left\langle\partial_{t} \rho^{(n)}, \rho^{(n)}\right\rangle
$$

where the brackets stands for the duality product between $\left\{\rho\right.$ such that $\left.\nabla_{x} \rho \in \mathcal{H}\right\}$ and $\left\{\sum_{|\alpha|=1} \partial_{x}^{\alpha} J, J \in \mathcal{H}^{\prime}\right\}$. Passing to the limit $n \rightarrow \infty$ justifies that

$$
\frac{d}{d t}\|\rho\|_{L^{2}}=2\left\langle\partial_{t} \rho, \rho\right\rangle
$$

holds in $\mathcal{D}^{\prime}(] 0, T[)$, which gives (32). Therefore, one deduces that $\frac{d}{d t}\|\rho\|_{L^{2}} \in L^{1}(0, T)$ and

$$
\|\rho(t)\|_{L^{2}}^{2}=\left\|\rho_{I}\right\|_{L^{2}}^{2}+\int_{0}^{t} 2\left\langle\partial_{t} \rho, \rho\right\rangle d s
$$

defines a continuous function on $[0, T]$. Furthermore,

$$
\left\|\rho(t)-\rho\left(t_{0}\right)\right\|_{L^{2}}^{2}=\|\rho(t)\|_{L^{2}}^{2}+\left\|\rho\left(t_{0}\right)\right\|_{L^{2}}^{2}-2\left(\rho(t), \rho\left(t_{0}\right)\right)_{L^{2}}
$$

tends to 0 as $t$ goes to $t_{0}$ since $\rho \in C^{0}\left([0, T], L^{2}-\right.$ weak $)$.

In turn, one deduces the following uniqueness statement which proves that the whole sequence $\rho^{\eta}$ converges.

Corollary 5 There exists a unique solution $\rho$ of (21) with $\rho \in L^{\infty}\left(\mathbb{R}^{+} ; L^{2}\left(\mathbb{R}^{N} \times \mathbb{N}, B_{i}^{-1}\right.\right.$ di® $d x))$ and $\nabla_{x} \rho \in L^{2}\left(\mathbb{R}^{+} ; \mathcal{H}\right)$.

Proof. The previous Lemma guarantees that $\rho$ is continuous with values in $L^{2}\left(\mathbb{R}^{N} \times\right.$ $\left.\mathbb{N}, B_{i}^{-1} d i \otimes d x\right)$. By linearity, it suffices to consider the solution $\rho$ corresponding to data $\rho_{I}=0$. Then, (32) combined to the positivity of $\mathcal{D}$ implies that $\|\rho(t)\|_{L^{2}}=0$.

As a concluding remark, let us notice that we can improve the convergence of $\rho^{\eta}$ to $\rho$. Indeed, using $\omega \delta_{i j}$ as test function in (26), we obtain that $\nabla_{x} \rho_{i}$ reads as a bounded term 
in $L^{2}\left((0, T) \times \mathbb{R}^{N}\right)$ plus $\eta \times\left(\right.$ first derivatives of bounded terms in $\left.\left.L^{2}\left((0, T) \times \mathbb{R}^{N}\right)\right)\right)$, hence it is compact in $H^{-1}((0, T) \times B(0, R))$ for any $0<T, R<\infty$. Combining this information to the continuity equation in Lemma 3, we can apply the Div-Curl argument of L. Tartar [39], as in [34], [29], [20], [28] and we deduce that for any $i \in \mathbb{N}, \rho_{i}^{\eta}$ lies in a compact set of $L_{\text {loc }}^{2}\left((0, T) \times \mathbb{R}^{N}\right)$. If further the $B_{i}$ 's tend to 0 , one easily concludes that $\rho^{\eta}$ converges to $\rho$ strongly in $L^{2}((0, T) \times B(0, R) \times \mathbb{N})$, for any $0<T, R<\infty$.

\section{A Properties of the collision operators}

\section{A.1 Proof of Proposition 1}

We write $Q_{0}^{e l}(f)_{i}=K(f)_{i}-\Sigma_{i} f_{i}$. Of course, one has $\|\Sigma f\|_{\mathbb{F}}=\|f\|_{\mathbb{E}}$. Next, using (h1), we get

$$
\begin{aligned}
& \sum_{i} \int_{S^{N-1}}\left|K(f)_{i}\right|^{2} \frac{1}{\Sigma_{i} B_{i}} d \omega= \\
& =\sum_{i} \int_{S^{N-1}}\left|\int_{S^{N-1}} \Gamma_{i}\left(\omega^{\prime}, \omega\right) f_{i}\left(\omega^{\prime}\right) d \omega^{\prime}\right|^{2} \frac{1}{\Sigma_{i} B_{i}} d \omega \\
& \leq \sum_{i} \int_{S^{N-1}}\left\{\left(\int_{S^{N-1}} \Gamma_{i}\left(\omega^{\prime}, \omega\right) d \omega\right)\left|f_{i}\left(\omega^{\prime}\right)\right|^{2} d \omega^{\prime} \quad \int_{S^{N-1}} \Gamma_{i}\left(\omega, \omega^{\prime}\right) d \omega^{\prime} \frac{1}{\Sigma_{i} B_{i}}\right\} d \omega \\
& \leq \sum_{i} \int_{S^{N-1}} \Sigma_{i}\left(\omega^{\prime}\right)\left|f_{i}\left(\omega^{\prime}\right)\right|^{2} B_{i}^{-1} d \omega^{\prime}=\|f\|_{\mathbb{E}}^{2} .
\end{aligned}
$$

It follows that $Q_{0}^{e l} \in \mathcal{L}(\mathbb{E}, \mathbb{F})$, with $\left\|Q_{0}^{e l}(f)\right\|_{\mathbb{F}} \leq 2\|f\|_{\mathbb{E}}$. According to Hypothesis (h1), we easily get $(i)$

$$
\int_{S^{N-1}} Q_{0}^{e l}(f)_{i} d \omega=\int_{S^{N-1}} f_{i}(\omega)\left(\int_{S^{N-1}}\left(\Gamma_{i}\left(\omega, \omega^{\prime}\right)-\Gamma_{i}\left(\omega^{\prime}, \omega\right)\right) d \omega^{\prime}\right) d \omega=0 .
$$

INRIA 
Now, $\mathcal{B}_{0}$ is clearly bilinear and continuous on $\mathbb{E} \times \mathbb{E}$ and we compute

$$
\begin{aligned}
\mathcal{B}_{0}(f, f)= & -\sum_{i} \int_{S^{N-1}} \int_{S^{N-1}} \Gamma_{i}\left(\omega^{\prime}, \omega\right)\left[f_{i}\left(\omega^{\prime}\right)-f_{i}(\omega)\right] f_{i}(\omega) B_{i}^{-1} d \omega^{\prime} d \omega \\
= & -\sum_{i} \int_{S^{N-1}} \int_{S^{N-1}} \Gamma_{i}\left(\omega^{\prime}, \omega\right) f_{i}(\omega) f_{i}\left(\omega^{\prime}\right) B_{i}^{-1} d \omega^{\prime} d \omega \\
& +\frac{1}{2} \sum_{i} \int_{S^{N-1}} \int_{S^{N-1}} \Gamma_{i}\left(\omega^{\prime}, \omega\right)\left|f_{i}(\omega)\right|^{2} d \omega^{\prime} d \omega \\
& +\frac{1}{2} \sum_{i} \int_{S^{N-1}} \int_{S^{N-1}} \Gamma_{i}\left(\omega, \omega^{\prime}\right)\left|f_{i}\left(\omega^{\prime}\right)\right|^{2} d \omega^{\prime} d \omega \\
= & 1 / 2 \sum_{i} \int_{S^{N-1}} \int_{S^{N-1}} \Gamma_{i}\left(\omega^{\prime}, \omega\right)\left(\left|f_{i}(\omega)\right|^{2}+\left|f_{i}\left(\omega^{\prime}\right)\right|^{2}\right. \\
= & \left.1 / 2 \sum_{i} \int_{S^{N-1}} \int_{S^{N-1}} \Gamma_{i}\left(\omega^{\prime}, \omega\right) \mid f_{i}(\omega)-f_{i}(\omega) f_{i}\left(\omega^{\prime}\right)\right) B^{-1}{ }^{-1} B_{i}^{-1} d \omega^{\prime} d \omega
\end{aligned}
$$

This obviously leads to the characterization of the kernel of $Q_{0}^{e l}$ in (iii).

Moreover, we have

$$
\begin{aligned}
& \sum_{i} \int_{S^{N-1}}\left|Q_{0}^{e l}(f)_{i}\right|^{2} \frac{1}{\Sigma_{i} B_{i}} d \omega \\
& \leq \sum_{i} \int_{S^{N-1}} \int_{S^{N-1}} \Gamma_{i}\left(\omega^{\prime}, \omega\right)\left|f_{i}(\omega)-f_{i}\left(\omega^{\prime}\right)\right|^{2} \frac{\Sigma_{i}}{\Sigma_{i} B_{i}} d \omega^{\prime} d \omega \leq 2 \mathcal{B}_{0}(f, f),
\end{aligned}
$$

which implies the last inequality in assertion (ii).

\section{A.2 Proof of Corollary 1}

Set $f=\langle f\rangle-r$. Since $r$ has null average, Assumption (h2) yields

$$
\begin{aligned}
\mathcal{B}_{0}(f, f) & =1 / 2 \sum_{i} \int_{S^{N-1}} \int_{S^{N-1}} \Gamma_{i}\left(\omega^{\prime} \omega\right)\left|f_{i}\left(\omega^{\prime}\right)-f_{i}(\omega)\right|^{2} B_{i}^{-1} d \omega^{\prime} d \omega \\
& \geq 1 / 2 \sum_{i} \int_{S^{N-1}} \int_{S^{N-1}}\left|f_{i}\left(\omega^{\prime}\right)-f_{i}(\omega)\right|^{2} \gamma_{i} B_{i}^{-1} d \omega^{\prime} d \omega \\
& \geq 1 / 2 \sum_{i} \int_{S^{N-1}} \int_{S^{N-1}}\left|r_{i}\left(\omega^{\prime}\right)-r_{i}(\omega)\right|^{2} \gamma_{i} B_{i}^{-1} d \omega^{\prime} d \omega \\
& \geq 1 / 2 \sum_{i} \int_{S^{N-1}} \int_{S^{N-1}}\left(r_{i}^{2}\left(\omega^{\prime}\right)+r_{i}^{2}(\omega)-2 r_{i}(\omega) r_{i}\left(\omega^{\prime}\right)\right) \gamma_{i} B_{i}^{-1} d \omega^{\prime} d \omega \\
& \geq \sum_{i} \int_{S^{N-1}} r_{i}^{2}(\omega) \gamma_{i} B_{i}^{-1} d \omega=N^{2}(r) .
\end{aligned}
$$

RR $\mathrm{n}^{\circ} 4302$ 


\section{A.3 Proof of Proposition 2}

First, let us estimate

$$
\begin{aligned}
\left\|Q_{1}^{e l}(f)\right\|_{\mathbb{F}}^{2} & =\sum_{i} \int_{S^{N-1}}\left|\sum_{j} \int_{S^{N-1}} \sigma_{i j}\left(\omega, \omega^{\prime}\right)\left(f_{j}\left(\omega^{\prime}\right)-f_{j}(\omega)\right) d \omega^{\prime}\right|^{2}\left(\Sigma_{i}(\omega) B_{i}\right)^{-1} d \omega \\
\leq & \sum_{i} \int_{S^{N-1}}\left(\sum_{j} \int_{S^{N-1}} \sigma_{i j}\left(\omega, \omega^{\prime}\right) B_{j} d \omega^{\prime}\right. \\
& \left.\times \sum_{j} \int_{S^{N-1}} \sigma_{i j}\left(\omega, \omega^{\prime}\right)\left|f_{j}\left(\omega^{\prime}\right)-f_{j}(\omega)\right|^{2} B_{j}^{-1} d \omega^{\prime}\right)\left(\Sigma_{i} B_{i}\right)^{-1} d \omega \\
\leq & M_{2} \sum_{j} \int_{S^{N-1}} \int_{S^{N-1}}\left(\sum_{i} \sigma_{i j}\left(\omega, \omega^{\prime}\right)\right)\left|f_{j}\left(\omega^{\prime}\right)-f_{j}(\omega)\right|^{2} B_{j}^{-1} d \omega^{\prime} d \omega \\
\leq & M_{2} \sum_{j} \int_{S^{N-1}} \int_{S^{N-1}} \Gamma_{j}\left(\omega, \omega^{\prime}\right)\left|f_{j}\left(\omega^{\prime}\right)-f_{j}(\omega)\right|^{2} B_{j}^{-1} d \omega^{\prime} d \omega \\
\leq & 2 M_{2} \mathcal{B}_{0}(f, f) \leq 2 M_{2}\left\|Q_{0}^{e l}(f)\right\|_{\mathbb{F}}\|f\|_{\mathbb{E}} \leq 4 M_{2}\|f\|_{\mathbb{E}}^{2}
\end{aligned}
$$

where we used the Cauchy-Schwarz inequality and the results in Proposition 1 . This proves that $Q_{1}^{e l} \in \mathcal{L}(\mathbb{E}, \mathbb{F})$.

In view of Remark 2, we can integrate $Q_{1}^{e l}$ with respect to $\omega$ and we get

$$
\begin{aligned}
\int_{S^{N-1}} Q_{1}^{e l}(f)_{i} d \omega & =\sum_{j} \int_{S^{N-1}} f_{j}\left(\omega^{\prime}\right)\left(\int_{S^{N-1}} \sigma_{i j}\left(\omega, \omega^{\prime}\right) d \omega\right) d \omega^{\prime} \\
& -\sum_{j} \int_{S^{N-1}} f_{j}(\omega)\left(\int_{S^{N-1}} \sigma_{i j}\left(\omega, \omega^{\prime}\right) d \omega^{\prime}\right) d \omega \\
& =\sum_{j} \int_{S^{N-1}} f_{j}(\omega)\left(\int_{S^{N-1}}\left(\sigma_{i j}\left(\omega^{\prime}, \omega\right)-\sigma_{i j}\left(\omega, \omega^{\prime}\right)\right) d \omega^{\prime}\right) d \omega \\
& =0,
\end{aligned}
$$

by using the symmetry assumption (h1').

Then, $\mathcal{B}_{1}$ is obviously a bilinear continuous form on $\mathbb{E}$, and a straightforward computation yields

$$
\begin{aligned}
\mathcal{B}_{1}(f, g)= & -\sum_{i, j} \int_{S^{N-1}} \int_{S^{N-1}} \sigma_{i j}\left(\omega, \omega^{\prime}\right)\left(f_{j}\left(\omega^{\prime}\right)-f_{j}(\omega)\right) g_{i}(\omega) B_{i}^{-1} d \omega^{\prime} d \omega \\
= & \sum_{i, j} \int_{S^{N-1}} \int_{S^{N-1}} \sigma_{i j}\left(\omega^{\prime}, \omega\right)\left(f_{j}\left(\omega^{\prime}\right)-f_{j}(\omega)\right) g_{i}\left(\omega^{\prime}\right) B_{i}^{-1} d \omega d \omega^{\prime} \\
= & 1 / 2 \sum_{i, j} \int_{S^{N-1}} \int_{S^{N-1}}\left(f_{j}\left(\omega^{\prime}\right)-f_{j}(\omega)\right) \\
& \left(\sigma_{i j}\left(\omega^{\prime}, \omega\right) g_{i}\left(\omega^{\prime}\right)-\sigma_{i j}\left(\omega, \omega^{\prime}\right) g_{i}(\omega)\right) B_{i}^{-1} d \omega^{\prime} d \omega
\end{aligned}
$$

INRIA 
Finally, in view of (10), it is easy to check that $\mathcal{E}_{0} \subset \operatorname{Ker}\left(Q_{1}^{e l}\right)$, and writing $f=\langle f\rangle+r$, we immediately get

$$
\begin{aligned}
\left|\mathcal{B}_{1}(f, f)\right|=\left|\left(Q_{1}^{e l}(f), f\right)_{\mathbb{L}}\right|=\left|\left(Q_{1}^{e l}(r), r\right)_{\mathbb{L}}\right| & \leq\|r\|_{\mathbb{E}}\left\|Q_{1}^{e l}(r)\right\|_{\mathbb{F}} \\
& \leq 2 \sqrt{M_{2}}\|r\|_{\mathbb{E}}^{2} \\
& \leq 2 M_{0} \sqrt{M_{2}} N(f-\langle f\rangle)^{2} .
\end{aligned}
$$

We can improve this bound, by noticing that, since the average of $r$ vanishes, (34) and (h3) yield

$$
\begin{aligned}
\left\|Q_{1}^{e l}(r)\right\|_{\mathbb{F}}^{2} & \leq M_{0} M_{2} \sum_{j} \gamma_{j} B_{j}^{-1} \int_{S^{N-1}} \int_{S^{N-1}}\left|r_{j}\left(\omega^{\prime}\right)-r_{j}(\omega)\right|^{2} d \omega^{\prime} d \omega \\
& \leq M_{0} M_{2} \sum_{j} \gamma_{j} B_{j}^{-1}\left(2\left\langle r_{j}^{2}\right\rangle-2\left\langle r_{j}\right\rangle^{2}\right) \\
& \leq 2 M_{0} M_{2} \sum_{j} \gamma_{j} B_{j}^{-1}\left\langle r_{j}^{2}\right\rangle=2 M_{0} M_{2} N(r)^{2}
\end{aligned}
$$

and therefore

$$
\mathcal{B}_{1}(f, f) \leq \sqrt{2 M_{0} M_{2}}\|r\|_{\mathbb{E}} N(r) \leq M_{0} \sqrt{2 M_{2}} N(f-\langle f\rangle)^{2} .
$$

\section{A.4 Proof of Corollary 2.}

By combining the symmetry condition (h1") with estimate (h2"), we can dominate $\left|\mathcal{B}_{1}(f, f)\right|$, as given in Proposition 2-ii) by

$$
\begin{gathered}
M / 2 \sum_{i j} \int_{S^{N-1}} \int_{S^{N-1}} \gamma_{i} \gamma_{j}\left|f_{j}\left(\omega^{\prime}\right)-f_{j}(\omega)\right|\left|f_{i}\left(\omega^{\prime}\right)-f_{i}(\omega)\right| d \omega d \omega^{\prime} \\
\leq M / 2 \sum_{i j} \gamma_{i} \gamma_{j}\left(\int_{S^{N-1}} \int_{S^{N-1}}\left|f_{j}\left(\omega^{\prime}\right)-f_{j}(\omega)\right|^{2} d \omega d \omega^{\prime}\right)^{1 / 2} \\
\times\left(\int_{S^{N-1}} \int_{S^{N-1}}\left|f_{i}\left(\omega^{\prime}\right)-f_{i}(\omega)\right|^{2} d \omega d \omega^{\prime}\right)^{1 / 2} .
\end{gathered}
$$

Since one assumes $\langle f\rangle=0$, the integral becomes

$$
\int_{S^{N-1}} \int_{S^{N-1}}\left|f_{i}\left(\omega^{\prime}\right)-f_{i}(\omega)\right|^{2} d \omega d \omega^{\prime}=2\left\langle f_{i}^{2}\right\rangle .
$$

$\mathrm{RR} \mathrm{n}^{\circ} 4302$ 
Hence, we get

$$
\begin{aligned}
\left|\mathcal{B}_{1}(f, f)\right| & \leq M \sum_{i j} \gamma_{i} \gamma_{j}\left\langle f_{j}^{2}\right\rangle^{1 / 2}\left\langle f_{i}^{2}\right\rangle^{1 / 2} \\
& \leq M\left(\sum_{i j} \gamma_{i} B_{i}^{-1}\left\langle f_{i}^{2}\right\rangle \gamma_{j} B_{j}^{-1}\left\langle f_{j}^{2}\right\rangle\right)^{1 / 2}\left(\sum_{i j} \gamma_{i} B_{i} \gamma_{j} B_{j}\right)^{1 / 2} \\
& \leq M M_{1} \sum_{i} \gamma_{i} B_{i}^{-1}\left\langle f_{i}^{2}\right\rangle=M M_{1} N(f)^{2} .
\end{aligned}
$$

$\square$ 


\section{References}

[1] Allaire G. and Bal G., Homogeneization of the criticality spectral equation in neutron transport, Math. Model. Numer. Anal., 33, 721-746 (1999); Announced in Homogénéisation d'une équation spectrale du transport neutronique, CRAS, 325, 1043-1048 (1997).

[2] Allaire G. and CapdeboscQ Y., Homogeneization of a spectral problem for a multigroup neutronic diffusion model, Comput. Methods Appl. Mech. Engrg. 187, 91-117 (2000).

[3] Allaire G. and Malige F., Analyse asymptotique spectrale d'un problème de diffusion neutronique, CRAS, 324, 939-944 (1997).

[4] BAL G., Couplage d'équations et homogénéisation en transport neutronique, Thèse de doctorat de l'Université Paris 6, 1997.

[5] Bardos C., Dumas L., Gérard P. and Golse F., Systèmes dynamiques et équations cinétiques: résultats et perspectives in Les grands systèmes des sciences et de la technologie (en hommage à R. Dautray), J. Horowitz and J.L. Lions Eds., RMA Res. Notes Appl. Math., 28, pp. 49-62 (Masson, 1994)

[6] Bardos C., Golse F. and Perthame B., The Rosseland approximation for the radiative transfer equations, CPAM, 40, 691-721 (1987) and CPAM, 42, 891-894 (1989).

[7] Bardos C., Golse F., Perthame B. and Sentis R., The nonaccretive radiative transfer equations: existence of solutions ans Rosseland approximations, J. Funct. Anal., 77, 434460 (1988).

[8] Bardos C., Santos R. and Sentis R., Diffusion approximation and computation of the critical size, Trans. Am. Math. Soc., 284, 617-649 (1984).

[9] Ben Abdallah N. and Degond P., On a hierarchy of macroscopic models for semiconductors, J. Math. Phys., 37, no. 7, 3306-3333 (1996).

[10] Bensoussan A., Lions J.-L. and Papanicolaou G., Boundary layers and homogeneization of transport processes, Publ. RIMS Kyoto Univ., 15, 53-157 (1979).

[11] Bourgade J.P., On Spherical Harmonics Expansion type models for electron-phonon collisions, Preprint.

[12] Bussac J. and Reuss P., Traité de neutronique (Herrman, 1978).

[13] Capdeboscq Y., Homogénéisation des modèles de diffusion en neutronique, Thèse de l'Université Paris 6, 2000.

[14] Capdeboscq Y., Homogenization of a spectral problem with drift, Preprint 2000 and Homogenization of a diffusion equation with drift, CRAS, 327, 807-812 (2000). 
[15] Cercignani C., The Boltzmann equation and its applications, Applied Mathematical Sciences, vol. 67 (Springer-Verlag, 1988).

[16] Dautray R. and Lions J.-L., Analyse mathématique et calcul numérique pour les sciences et les techniques, vol. 1-3 (Masson, 1985).

[17] Davison B., Neutron transport theory (Oxford Clarendon Press, 1958).

[18] Degond P., Mathematical modelling of microelectronics semiconductor devices, in Some current topics on nonlinear conservation laws, Proc. of the Morningside Math. Center, pp. 77-110, AMS/IP Stud. Adv. Math., 15 (Amer. Math. Soc., 2000).

[19] Degond P., An infinite system of diffusion equations arising in transport theory: the coupled Spherical Harmonics Expansion model, to appear in M3AS.

[20] Degond P., Goudon T. and Poupaud F., Diffusion approximation for non homogeneous and non microreversible processes, Indiana Univ. Math. J., 49, 1175-1198, 2000.

[21] Dorning J., Uddin R. and Zhang H., Systematic homogenization and self-consistent flux and pin power reconstruction for nodal diffusion-I: Diffusion equation-based theory, Nucl. Sci. Eng., 121, 226-244 (1995).

[22] Dumas L., and Golse F., Homogenization of transport equations, SIAM J. Appl. Math., $60,1447-1470(2000)$.

[23] Gérard P. and Golse F., Averaging regularity results for pdes under transversality assumptions, Comm. Pure Appl. Math., 45, 1-26, 1992.

[24] Golse F., Remarques sur l'homogénéisation des équations de transport, CRAS, 305, 801-804 (1987).

[25] Golse F., Particle transport in nonhomogeneous media, in Mathematical aspects of Fluids and Plasma Dynamics, Proc. Salice Terme 1988, Boffi-Rionero-Toscani Eds., Lect. Notes Math, vol. 1460, pp. 152-170 (Springer, 1991)

[26] Golse F., From kinetic to macroscopic models, Session "Etat de la Recherche" SMF, Orléans, 4-6 juin 1998.

[27] Golse F. and Poupaud F., Limite fluide des équations de Boltzmann des semiconducteurs pour une statistique de Fermi-Dirac, Asymptotic Analysis, 6, 135-160 (1992).

[28] Goudon T. and Mellet A., Diffusion approximation in heterogeneous media, to appear in Asymptotic Analysis.

[29] Goudon T. and Poupaud F., Approximation by homogeneization and diffusion of kinetic equations, Comm. Partial Differential Equations, 26, 537-569 (2001). 
[30] Larsen E., Neutron transport and diffusion in inhomogeneous media, I, J. Math. Phys., $16,1421-1427(1975)$.

[31] LaRsen E., Neutron transport and diffusion in inhomogeneous media, II, Nuclear Sc. and Engineering., 60, 357-368 (1976).

[32] Larsen E. and Keller J., Asymptotic solution of neutron transport processes for small free paths, J. Math. Phys., 15, 75-81 (1974).

[33] Larsen E. and Williams M., Neutron drift in heterogeneous media, Nuclear Sc. and Engrg., 65, 290-302 (1978).

[34] Lions P.-L. and Toscani G., Diffuse limit for finite velocity Boltzmann kinetic models, Rev. Mat. Ib., 13, 473-513 (1997).

[35] Malvagi F., Levermore C. and Pomraning G., Asymptotics limits of a statistical transport description, in Modern Math. Methods in Transport Theory, W. Greenberg-J. Polewczak Eds., Op. Theory : Adv. and Appl., vol. 51, pp. 200-207 (Birkhauser, 1991).

[36] Petterson R., Existence theorems for the linear, space-inhomogeneous transport equation, IMA J. Appl. Math., 30, 81-105 (1983).

[37] Planchard J., Méthodes mathématiques en neutronique, Coll. Direction des Etudes et Recherches d'Electricité de France, vol. 90 (Eyrolles, 1995).

[38] Poupaud F., Diffusion approximation of the linear semiconductor Boltzmann equation: analysis of boundary layers, Asymptotic Analysis, 4, 293-317 (1991).

[39] Tartar L., Compensated compactness and applications to pde, in Herriot-Watt Symp., vol. IV, R. Knopps Eds., Res. Notes in Math, vol. 39, pp. 136-212 (Pitman, 1979).

[40] WaChSPRess E., Iterative solution of elliptic systems and application to the neutron diffusion equations of reactor physics (Practice-Hall, 1966).

[41] Wigner E., Nuclear reactor theory (AMS, 1961).

$\mathrm{RR} \mathrm{n}^{\circ} 4302$ 


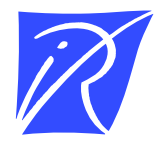

Unité de recherche INRIA Sophia Antipolis 2004, route des Lucioles - BP 93 - 06902 Sophia Antipolis Cedex (France)

Unité de recherche INRIA Lorraine : LORIA, Technopôle de Nancy-Brabois - Campus scientifique 615, rue du Jardin Botanique - BP 101 - 54602 Villers-lès-Nancy Cedex (France)

Unité de recherche INRIA Rennes : IRISA, Campus universitaire de Beaulieu - 35042 Rennes Cedex (France)

Unité de recherche INRIA Rhône-Alpes : 655, avenue de l'Europe - 38330 Montbonnot-St-Martin (France)

Unité de recherche INRIA Rocquencourt : Domaine de Voluceau - Rocquencourt - BP 105 - 78153 Le Chesnay Cedex (France)

Éditeur

INRIA - Domaine de Voluceau - Rocquencourt, BP 105 - 78153 Le Chesnay Cedex (France)

http://www.inria.fr

ISSN 0249-6399 